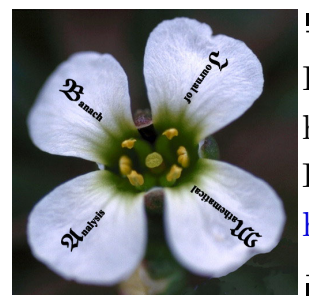

Banach J. Math. Anal. 9 (2015), no. 3, 206-227

http://doi.org/10.15352/bjma/09-3-15

ISSN: $1735-8787$ (electronic)

http://projecteuclid.org/bjma

\title{
COMPACTNESS OF COMMUTATORS AND MAXIMAL COMMUTATORS OF MULTILINEAR SINGULAR INTEGRAL OPERATORS WITH NON-SMOOTH KERNELS ON MORREY SPACE
}

\author{
RUI BU ${ }^{1}$, XIAOLI GUO ${ }^{2 *}$ AND QIANG HUANG ${ }^{1}$
}

Communicated by M. A. Ragusa

\begin{abstract}
In this paper, the behavior for commutators and maximal commutators of a class of bilinear singular integral operators associated with nonsmooth kernels on the products of Morrey space is studied. By some maximal operators and commutators, we proved that the commutators and maximal commutators of singular integral operators and CMO functions are bounded and compact.
\end{abstract}

\section{INTRODUCTION}

In recent years, considerable attention has been paid to the study of multilinear singular integral. In this article, we will address the m-linear operators $T$ which defined on the m-fold product of Schwartz spaces and taking values into the space of tempered distributions,

$$
T: \mathcal{S}\left(\mathbb{R}^{n}\right) \times \cdots \times \mathcal{S}\left(\mathbb{R}^{n}\right) \mapsto \mathcal{S}^{\prime}\left(\mathbb{R}^{n}\right),
$$

and associated with kernel $K\left(x, y_{1}, \ldots, y_{m}\right)$ in the sense that

$$
T\left(f_{1}, \ldots, f_{m}\right)(x)=\int_{\left(\mathbb{R}^{n}\right)^{m}} K\left(x, y_{1}, \ldots, y_{m}\right) f_{1}\left(y_{1}\right) \cdots f_{m}\left(y_{m}\right) \mathrm{d} y_{1} \cdots \mathrm{d} y_{m},
$$

Date: Received: Jul, 24, 2014; Accepted: Nov. 5, 2014.

* Corresponding author.

2010 Mathematics Subject Classification. Primary 42B20; Secondary 42B25, 47B07.

Key words and phrases. Morrey space, singular integral operator, maximal operator, commutator, compactness. 
where $K\left(x, y_{1}, \ldots, y_{m}\right)$ is a locally integral function defined away from the diagonal $x=y_{1}=\cdots=y_{m}$ in $\left(\mathbb{R}^{n}\right)^{m+1}, x \notin \cap_{j=1}^{m} \operatorname{supp} f_{j}$ and $f_{1}, \ldots, f_{m}$ are bounded functions with compact supports. The corresponding maximal singular integral operator $T^{*}$ is defined by

$$
T^{*}\left(f_{1}, \cdots, f_{m}\right)(x)=\sup _{\delta>0}\left|T_{\delta}\left(f_{1}, \cdots, f_{m}\right)(x)\right|,
$$

where

$$
T_{\delta}\left(f_{1}, \cdots, f_{m}\right)(x)=\int_{\left|x-y_{1}\right|^{2}+\cdots+\left|x-y_{m}\right|^{2}>\delta} K\left(x, y_{1}, \ldots, y_{m}\right) \prod_{i=1}^{m} f_{i}\left(y_{i}\right) \mathrm{d} y_{1} \cdots \mathrm{d} y_{m} .
$$

A kernel $K$ is called of class $m-C Z K(A, \gamma)$ if there exist positive constants $A$ and $\gamma \in(0,1]$ such that $K$ satisfies the size condition

$$
\left|K\left(x, y_{1}, \ldots, y_{m}\right)\right| \leq \frac{A}{\left(\left|x-y_{1}\right|+\cdots+\left|x-y_{m}\right|\right)^{m n}},
$$

for all $\left(x, y_{1}, \ldots, y_{m}\right) \in\left(\mathbb{R}^{n}\right)^{m+1}$ with $x \neq y_{j}$ for some $j \in\{1,2, \ldots, m\}$; and the smoothness condition

$$
\left|K\left(x, y_{1}, \ldots, y_{j}, \ldots, y_{m}\right)-K\left(x^{\prime}, y_{1}, \ldots, y_{j}, \ldots, y_{m}\right)\right| \leq \frac{A\left|x-x^{\prime}\right|^{\gamma}}{\left(\sum_{i=1}^{m}\left|x-y_{i}\right|\right)^{m n+\gamma}},
$$

whenever $\left|x-x^{\prime}\right| \leq \frac{1}{2} \max _{1 \leq j \leq m}\left|x-y_{j}\right|$, and also for each $j$,

$$
\left|K\left(x, y_{1}, \ldots, y_{j}, \ldots, y_{m}\right)-K\left(x, y_{1}, \ldots, y_{j}^{\prime}, \ldots, y_{m}\right)\right| \leq \frac{A\left|y_{j}-y_{j}^{\prime}\right|^{\gamma}}{\left(\sum_{i=1}^{m}\left|x-y_{i}\right|\right)^{m n+\gamma}}
$$

whenever $\left|y_{j}-y_{j}^{\prime}\right| \leq \frac{1}{2} \max _{1 \leq j \leq m}\left|x-y_{j}\right|$. Then the operator $T$ associated with this kernel $K$ is called the multilinear Calderón-Zygmund operator. For the multilinear Calderón-Zygmund operator T, in [17], Grafakos and Torres obtained the multilinear $T 1$ theorem. Thus they show the boundedness of $T$ on the products of Lebesgue spaces. Moreover, in [16], the weighted estimates with $A_{p}$ weights for the operators $T$ and $T^{*}$ was considered. For more works about multilinear Calderón-Zygmund operator, the reader can refer to [16], [17], [21], [23] and the references therein.

In this article, we are interested in the commutators and the maximal commutators of multilinear singular integral operators. For the sake of convenience, we will only consider the bilinear setting, since the other multilinear case are quite similar.

For $b \in \operatorname{BMO}\left(\mathbb{R}^{n}\right)$, we define the following commutators

$$
\begin{aligned}
& T_{b, 1}\left(f_{1}, f_{2}\right)(x)=[b, T]_{1}\left(f_{1}, f_{2}\right)(x)=T\left(b f_{1}, f_{2}\right)(x)-b T\left(f_{1}, f_{2}\right)(x), \\
& T_{b, 2}\left(f_{1}, f_{2}\right)(x)=[b, T]_{2}\left(f_{1}, f_{2}\right)(x)=T\left(f_{1}, b f_{2}\right)(x)-b T\left(f_{1}, f_{2}\right)(x), \\
& T_{b, 1}^{*}\left(f_{1}, f_{2}\right)(x)=\sup _{\delta>0}\left|\left[b, T_{\delta}\right]_{1}\left(f_{1}, f_{2}\right)(x)\right|=\sup _{\delta>0}\left|\left(T_{\delta}\left(b f_{1}, f_{2}\right)-b T_{\delta}\left(f_{1}, f_{2}\right)\right)(x)\right|, \\
& T_{b, 2}^{*}\left(f_{1}, f_{2}\right)(x)=\sup _{\delta>0}\left|\left[b, T_{\delta}\right]_{2}\left(f_{1}, f_{2}\right)(x)\right|=\sup _{\delta>0}\left|\left(T_{\delta}\left(f_{1}, b f_{2}\right)-b T_{\delta}\left(f_{1}, f_{2}\right)\right)(x)\right| .
\end{aligned}
$$

Also, for a vector function

$$
\vec{b}=\left(b_{1}, b_{2}\right) \in \operatorname{BMO}\left(\mathbb{R}^{n}\right) \times \operatorname{BMO}\left(\mathbb{R}^{n}\right),
$$


we consider the following two iterated commutators introduced in [24],

$$
\begin{aligned}
& T_{\vec{b}}\left(f_{1}, f_{2}\right)(x)=\left[b_{2},\left[b_{1}, T\right]_{1}\right]_{2}\left(f_{1}, f_{2}\right)(x), \\
& T_{\vec{b}}^{*}\left(f_{1}, f_{2}\right)(x)=\sup _{\delta>0}\left|\left[b_{2},\left[b_{1}, T_{\delta}\right]_{1}\right]_{2}\left(f_{1}, f_{2}\right)(x)\right| .
\end{aligned}
$$

In the sense of (1.1), it is easy to see that

$$
\begin{aligned}
& T_{b, 1}\left(f_{1}, f_{2}\right)(x)=\int_{\mathbb{R}^{n}} \int_{\mathbb{R}^{n}} K\left(x, y_{1}, y_{2}\right)\left(b\left(y_{1}\right)-b(x)\right) f_{1}\left(y_{1}\right) f_{2}\left(y_{2}\right) \mathrm{d} y_{1} \mathrm{~d} y_{2}, \\
& T_{b, 2}\left(f_{1}, f_{2}\right)(x)=\int_{\mathbb{R}^{n}} \int_{\mathbb{R}^{n}} K\left(x, y_{1}, y_{2}\right)\left(b\left(y_{2}\right)-b(x)\right) f_{1}\left(y_{1}\right) f_{2}\left(y_{2}\right) \mathrm{d} y_{1} \mathrm{~d} y_{2}, \\
& T_{\vec{b}}\left(f_{1}, f_{2}\right)(x)=\int_{\mathbb{R}^{n}} \int_{\mathbb{R}^{n}} K\left(x, y_{1}, y_{2}\right)\left(b_{1}\left(y_{1}\right)-b_{1}(x)\right)\left(b_{2}\left(y_{2}\right)-b_{2}(x)\right) \prod_{i=1}^{2} f_{i}\left(y_{i}\right) \mathrm{d} \vec{y}, \\
& T_{b, 1}^{*}\left(f_{1}, f_{2}\right)(x)=\sup _{\delta>0}\left|\iint_{\left|x-y_{1}\right|^{2}+\left|x-y_{2}\right|^{2}>\delta} K\left(x, y_{1}, y_{2}\right)\left(b\left(y_{1}\right)-b(x)\right) \prod_{i=1}^{2} f_{i}\left(y_{i}\right) \mathrm{d} \vec{y}\right|, \\
& T_{b, 2}^{*}\left(f_{1}, f_{2}\right)(x)=\sup _{\delta>0}\left|\iint_{\left|x-y_{1}\right|^{2}+\left|x-y_{2}\right|^{2}>\delta} K\left(x, y_{1}, y_{2}\right)\left(b\left(y_{2}\right)-b(x)\right) \prod_{i=1}^{2} f_{i}\left(y_{i}\right) \mathrm{d} \vec{y}\right|, \\
& T_{\vec{b}}^{*}\left(f_{1}, f_{2}\right)(x)=\sup _{\delta>0}\left|\iint_{\sum_{i=1}^{2}\left|x-y_{i}\right|^{2}>\delta} K\left(x, y_{1}, y_{2}\right) \prod_{j=1}^{2}\left(b_{j}\left(y_{j}\right)-b_{j}(x)\right) \prod_{i=1}^{2} f_{i}\left(y_{i}\right) \mathrm{d} \vec{y}\right| .
\end{aligned}
$$

The commutator of multilinear Calderón-Zygmund operator has been extensively studied in last decades. To know the history of this topic, the reader can refer to [7], [23], [24], [25], [26], [28] and the references therein. Particularly, in [24], the boundedness of iterated commutators of multilinear Calderón-Zygmund operators on product of weighted Lebesgue spaces with multiple weights was studied, and in [28], the weighted strong and end-point estimates of maximal commutators of multilinear Calderón-Zygmund operators were considered.

However, for some multilinear singular integral operators including the Calderón commutator, people found that their kernels do not satisfy (1.3) (see [12]). Here, the Calderón commutator is defined by

$$
\mathcal{C}_{m+1}\left(f, a_{1}, \ldots, a_{m}\right)(x)=\int_{\mathbb{R}} \frac{\prod_{j=1}^{m}\left(A_{j}(x)-A_{j}(y)\right)}{(x-y)^{m+1}} f(y) \mathrm{d} y,
$$

where $A_{j}^{\prime}=a_{j}$. For this reason, in [12], via the generalized approximation to the identity, Duong et al. introduced a class of multilinear singular integral operators whose kernels satisfy certain "smoothness conditions" that are weaker than $K \in m-C Z K(A, \gamma)$.

For $p_{1}, \ldots, p_{m+1} \in[1, \infty]$ and $p \in(0, \infty)$ with $\frac{1}{p}=\sum_{j=1}^{m+1} \frac{1}{p_{j}}$, the following weak type estimate was established in [12],

$$
\left\|\mathcal{C}_{m+1}\left(f, a_{1}, \ldots, a_{m}\right)\right\|_{L^{p, \infty}(\mathbb{R})} \leq C\|f\|_{L^{p_{m+1}(\mathbb{R})}} \prod_{j=1}^{m}\left\|a_{j}\right\|_{L^{p_{k}(\mathbb{R})}}
$$


If $\min _{1 \leq j \leq m+1} p_{j}>1$, the strong type estimate was also established in [12]. Also, the weighted case, including the multiple weights, of the maximal Calderón commutator were considered in [11] and [15]. Moreover, there are a lot of works related to singular integral operators with non-smooth kernels. The reader may refer [11], [12], [13], [15], [18] and [19].

In this article, we are interested in the compactness of the commutator and maximal commutator of bilinear singular integral operators with non-smooth kernels and CMO functions on Morrey space, where CMO denotes the closure of $C_{c}^{\infty}$ in the BMO topology and $C_{c}^{\infty}$ is the set of $C^{\infty}$ functions with compact support. In particular, we will study the compactness on the Morrey space for the bilinear singular integral operator $T$ associated with a kernel $K$ which satisfies (1.2) in the sense (1.1), and

(1) $T$ is bounded from $L^{1}\left(\mathbb{R}^{n}\right) \times L^{1}\left(\mathbb{R}^{n}\right)$ to $L^{1 / 2, \infty}\left(\mathbb{R}^{n}\right)$;

(2) for $x, x^{\prime}, y_{1}, y_{2} \in \mathbb{R}^{n}$ with $8\left|x-x^{\prime}\right|<\min _{1 \leq j \leq 2}\left|x-y_{j}\right|$,

$$
\left|K\left(x, y_{1}, y_{2}\right)-K\left(x^{\prime}, y_{1}, y_{2}\right)\right| \leq \frac{C \tau^{\gamma}}{\left(\left|x-y_{1}\right|+\left|x-y_{2}\right|\right)^{2 n+\gamma}}
$$

where $C$ is a constant and $\tau$ is a number such that $2\left|x-x^{\prime}\right|<\tau$ and $4 \tau<$ $\min _{1 \leq j \leq 2}\left|x-y_{j}\right|$. It was pointed out in [19] that the kernel satisfying condition (1.2) and (1.4) includes the non-smooth kernel introduced by Doung et al. in [11], [12]. Hence, the study of the commutator and maximal commutator of bilinear singular integral operators with above non-smooth kernels is significative.

Before stating our results, we briefly describe the background and our motivation. The Morrey space $L^{p, \lambda}\left(\mathbb{R}^{n}\right)$ (see Definition 1.1) which was introduced by Morrey (see [22]) in 1938, is connected to certain problems in elliptic PDE. We note that in the linear setting, the compactness of commutator on Morrey space has been studied in [6]. Recently, Bényi and Torres posed a concept of compactness (see Definition 1.2) in the bilinear setting in [2] which was equivalent to the concept proposed by Calderón in [4]. Bényi and Torres then extended the result of compactness for linear singular integrals by Uchiyama [27] to the bilinear setting and obtained that $[b, T]_{1},[b, T]_{2},\left[b_{2},\left[b_{1}, T\right]_{1}\right]_{2}$ are compact bilinear operators from $L^{p_{1}}\left(\mathbb{R}^{n}\right) \times L^{p_{2}}\left(\mathbb{R}^{n}\right)$ to $L^{p}\left(\mathbb{R}^{n}\right)$ when $b, b_{1}, b_{2} \in \mathrm{CMO}\left(\mathbb{R}^{n}\right)$. Later, Ding et al. considered the compactness of the commutators of bilinear Calderón-Zygmund operators and CMO functions on the products of $L^{p, \lambda}\left(\mathbb{R}^{n}\right)$ space (see [10]), as well as the compactness of the maximal commutators of bilinear Calderón-Zygmund operators and CMO functions on the products of $L^{p}\left(\mathbb{R}^{n}\right)$ space (see [9]). We note that in [9] and [10], $T$ is a Calderón-Zygmund operator. In the article, we will consider the compactness of commutators and maximal commutators by assuming that $T$ is an operator associated with non-smooth kernel. This assumption raises essential difficulties if we following the proofs in [9] and [10].

To formulate the main hypotheses on the commutators under consideration, we need to define the John-Nirenberg space ([20]) of functions with Bounded Mean Oscillation (BMO). 
Let $f$ be a locally integrable function in $\mathbb{R}^{n}$. We say that $f$ belongs to BMO if

$$
\|f\|_{\mathrm{BMO}\left(\mathbb{R}^{\mathrm{n}}\right)}=\sup _{B \subset \mathbb{R}^{n}} \frac{1}{|B|} \int_{B}\left|f(x)-f_{B}\right| d x<\infty
$$

where $\mathrm{B}$ ranges in the class of balls of $\mathbb{R}^{n}$. Hereafter, $f_{B}$ stands for the integral average

$$
\frac{1}{|B|} \int_{B}|f(x)| d x
$$

of the function $f$ over the set B.

Definition 1.1. For $1 \leq p<\infty, n>1$ and $0<\lambda<n$, the Morrey space $L^{p, \lambda}\left(\mathbb{R}^{n}\right)$ is defined by

$$
L^{p, \lambda}\left(\mathbb{R}^{n}\right)=\left\{f \in L_{\mathrm{loc}}^{p}:\|f\|_{p, \lambda}=\sup _{y \in \mathbb{R}^{n}, r>0}\left(\frac{1}{r^{\lambda}} \int_{B(y, r)}|f(x)|^{p} \mathrm{~d} x\right)^{1 / p}<\infty\right\},
$$

where $B(y, r)$ denotes the ball centered at $y$ with radius $r>0$. The spaces $L^{p, \lambda}\left(\mathbb{R}^{n}\right)$ becomes a Banach space with norm $\|\cdot\|_{p, \lambda}$. If $\lambda=0$ and $\lambda=n$, then $L^{p, 0}\left(\mathbb{R}^{n}\right)$ and $L^{p, n}\left(\mathbb{R}^{n}\right)$ coincide with the space $L^{p}\left(\mathbb{R}^{n}\right)$ and $L^{\infty}\left(\mathbb{R}^{n}\right)$.

We denote the closed ball of radius $r$ centered at the origin in the normed space $X$ as $B_{r, X}=\{x \in X:\|x\| \leq r\}$.

Definition 1.2. A bilinear operator $T: X \times Y \mapsto Z$ is called compact if $T\left(B_{1, X} \times\right.$ $\left.B_{1, Y}\right)$ is precompact in $Z$.

Definition 1.3. A weight $w$ belongs to the class $A_{p}, 1<p<\infty$, if

$$
\sup _{Q}\left(\frac{1}{|Q|} \int_{Q} w(y) \mathrm{d} y\right)\left(\frac{1}{|Q|} \int_{Q} w(y)^{1-p^{\prime}} \mathrm{d} y\right)^{p-1}<\infty .
$$

A weight $w$ belongs to the class $A_{1}$ if there is a constant $C$ such that

$$
\frac{1}{|Q|} \int_{Q} w(y) \mathrm{d} y \leq C \inf _{x \in Q} w(x) .
$$

Definition 1.4. Let $\vec{p}=\left(p_{1}, p_{2}\right)$ and $1 / p=1 / p_{1}+1 / p_{2}$ with $1 \leq p_{1}, p_{2}<\infty$. Given $\vec{w}=\left(w_{1}, w_{2}\right)$, set $\nu_{\vec{w}}=\prod_{j=1}^{2} w_{j}^{p / p_{j}}$. We say that $\vec{w}$ satisfies the $A_{\vec{p}}$ condition if

$$
\sup _{Q}\left(\frac{1}{|Q|} \int_{Q} \nu_{\vec{w}}\right)^{1 / p} \prod_{j=1}^{2}\left(\frac{1}{|Q|} \int_{Q} w_{j}^{1-p_{j}^{\prime}}\right)^{1 / p_{j}^{\prime}}<\infty
$$

Here, $\left(\frac{1}{|Q|} \int_{Q} w_{j}^{1-p_{j}^{\prime}}\right)^{1 / p_{j}^{\prime}}$ is understood as $\left(\inf _{Q} w_{j}\right)^{-1}$, when $p_{j}=1$.

The following theorems are our main results:

Theorem 1.5. Let $T$ be a bilinear operator that bounded from $L^{1}\left(\mathbb{R}^{n}\right) \times L^{1}\left(\mathbb{R}^{n}\right)$ to $L^{1 / 2, \infty}\left(\mathbb{R}^{n}\right)$ and its kernel $K$ satisfies (1.2), (1.4). Assume $b \in \mathrm{CMO}\left(\mathbb{R}^{n}\right)$, $0<\lambda, \lambda_{1}, \lambda_{2}<n, p_{1}, p_{2} \in(1, \infty), p \in(1, \infty)$ such that $1 / p=1 / p_{1}+1 / p_{2}$ and $\lambda / p=\lambda_{1} / p_{1}+\lambda_{2} / p_{2}$. Then $T_{b, 1}^{*}, T_{b, 2}^{*}$ are compact from $L^{p_{1}, \lambda_{1}}\left(\mathbb{R}^{n}\right) \times L^{p_{2}, \lambda_{2}}\left(\mathbb{R}^{n}\right)$ to $L^{p, \lambda}\left(\mathbb{R}^{n}\right)$. 
Corollary 1.6. Let $T$ be a bilinear operator that bounded from $L^{1}\left(\mathbb{R}^{n}\right) \times L^{1}\left(\mathbb{R}^{n}\right)$ to $L^{1 / 2, \infty}\left(\mathbb{R}^{n}\right)$ and its kernel $K$ satisfies (1.2), (1.4). Assume $b \in \operatorname{CMO}\left(\mathbb{R}^{n}\right)$, $0<\lambda, \lambda_{1}, \lambda_{2}<n, p_{1}, p_{2} \in(1, \infty), p \in(1, \infty)$ such that $1 / p=1 / p_{1}+1 / p_{2}$ and $\lambda / p=\lambda_{1} / p_{1}+\lambda_{2} / p_{2}$. Then $T_{b, 1}, T_{b, 2}$ are compact from $L^{p_{1}, \lambda_{1}}\left(\mathbb{R}^{n}\right) \times L^{p_{2}, \lambda_{2}}\left(\mathbb{R}^{n}\right)$ to $L^{p, \lambda}\left(\mathbb{R}^{n}\right)$.

Theorem 1.7. Let $T$ be a bilinear operator that bounded from $L^{1}\left(\mathbb{R}^{n}\right) \times L^{1}\left(\mathbb{R}^{n}\right)$ to $L^{1 / 2, \infty}\left(\mathbb{R}^{n}\right)$ and its kernel $K$ satisfies (1.2), (1.4). Assume $b \in \operatorname{BMO}\left(\mathbb{R}^{n}\right)$, $0<\lambda, \lambda_{1}, \lambda_{2}<n, p_{1}, p_{2} \in(1, \infty), p \in(1, \infty)$ such that $1 / p=1 / p_{1}+1 / p_{2}$ and $\lambda / p=\lambda_{1} / p_{1}+\lambda_{2} / p_{2}$. Then

$$
\left\|T_{b, 1}^{*}\left(f_{1}, f_{2}\right)\right\|_{p, \lambda},\left\|T_{b, 2}^{*}\left(f_{1}, f_{2}\right)\right\|_{p, \lambda} \leq C\|b\|_{\mathrm{BMO}\left(\mathbb{R}^{n}\right)}\left\|f_{1}\right\|_{p_{1}, \lambda_{1}}\left\|f_{2}\right\|_{p_{2}, \lambda_{2}} .
$$

Remark 1.8. Theorem 1.5 and Corollary 1.6 are also true for the iterated commutators $T_{\vec{b}}^{*}$ and $T_{\vec{b}}$, and their proofs are similar to those of Theorem 1.5 and Corollary 1.6. We leave the detail to the interested reader. We will only write out the proof of Theorem 1.5, because the proof of Corollary 1.6 is quite similar. From [3] and Lemma 2.3, we can see that Theorem 1.7 is also true for the operators $T_{b, 1}\left(f_{1}, f_{2}\right)$ and $T_{b, 2}\left(f_{1}, f_{2}\right)$.

We make some conventions. In this paper, we always denote a positive constant by $C$ which is independent of the main parameters and its value may differ from line to line. We use the symbol $A \lesssim B$ to denote that there exists a constant $C$ such that $A \leq C B$. For a measurable set $E, \chi_{E}$ denotes its characteristic function. For a fixed $p$ with $p \in[1, \infty), p^{\prime}$ denotes the dual index of $p$. We also denote $\vec{f}=\left(f_{1}, \cdots, f_{m}\right)$ with scalar functions $f_{j}, j=1,2, \ldots, m$. Let $B(s, t)$ denote the ball centered at $s$ with radius $t>0$. Given $\alpha>0$ and a ball $B(s, t)$, $\alpha B(s, t)$ denotes the ball which is centered at $s$ with radius $\alpha t$. Let us define $f_{Q}$ as the average of $f$ over $\mathrm{Q}$ and $\mathrm{Q}$ is a cube in $\mathbb{R}^{n}$. Let $M$ be the standard Hardy-Littlewood maximal operator.

\section{Proof of TheOrem 1.7}

The proof of Theorem 1.7 needs some maximal functions in the following. For $0<\eta<\infty, M_{\eta}$ is the maximal operator

$$
M_{\eta} f(x)=M\left(|f|^{\eta}\right)^{1 / \eta}(x)=\left(\sup _{Q \ni x} \frac{1}{|Q|} \int_{Q}|f(y)|^{\eta} \mathrm{d} y\right)^{1 / \eta},
$$

and $M^{\#}$ is the sharp maximal operator defined by Fefferman and Stein [14],

$$
M^{\#} f(x)=\sup _{Q \ni x} \inf _{c} \frac{1}{|Q|} \int_{Q}|f(y)-c| \mathrm{d} y \approx \sup _{Q \ni x} \frac{1}{|Q|} \int_{Q}\left|f(y)-f_{Q}\right| \mathrm{d} y,
$$

and

$$
M_{\eta}^{\#} f(x)=M^{\#}\left(|f|^{\eta}\right)^{1 / \eta}(x)
$$

Moreover, when $0<p, \eta<\infty, w \in A_{\infty}\left(\mathbb{R}^{n}\right)$, then there exists $C>0$ such that

$$
\int_{\mathbb{R}^{n}}\left(M_{\eta} f(x)\right)^{p} w(x) \mathrm{d} x \leq C \int_{\mathbb{R}^{n}}\left(M_{\eta}^{\#} f(x)\right)^{p} w(x) \mathrm{d} x
$$

for every function $f$ for which the left-hand side is finite. 
Now let $b \in \operatorname{BMO}\left(\mathbb{R}^{n}\right)$, define the commutator $M_{b}$ of the Hardy-Littlewood maximal operator with $b$ by

$$
M_{b} f(x)=\sup _{B \ni x} \frac{1}{|B|} \int_{B}|b(x)-b(y)||f(y)| \mathrm{d} y
$$

for all $x \in \mathbb{R}^{n}$. For $\Phi(t)=t\left(1+\log ^{+} t\right)$ and a ball $B$ in $\mathbb{R}^{n}$,

$$
\|f\|_{L(\log L), B}=\inf \left\{\lambda>0: \frac{1}{|B|} \int_{B} \Phi\left(\frac{|f(x)|}{\lambda}\right) \mathrm{d} x \leq 1\right\} .
$$

Define the maximal operator $M_{L(\log L)}$ by

$$
M_{L(\log L)} f(x)=\sup _{B \ni x}\|f\|_{L(\log L), B}
$$

where the supremum is taken over all the balls containing $x$. By the generalized Jensen's inequlity, we have

$$
M_{L(\log L)} f(x) \lesssim M_{\eta} f(x)
$$

for any $\eta>1$.

Lemma 2.1. Let $b \in \mathrm{BMO}\left(\mathbb{R}^{n}\right)$, then for any $0<\eta<\frac{1}{2}$

$$
M_{\eta}^{\#}\left(M_{b} f\right)(x) \lesssim\|b\|_{\mathrm{BMO}\left(\mathbb{R}^{n}\right)} M_{L(\log L)} f(x),
$$

for any bounded functions $f$ with compact support.

This Lemma was proved in [1].

Furthermore, Grafakos, Liu, and Yang [15] introduced a kind of new bilinear maximal operator which was defined as:

$$
\begin{aligned}
& \mathcal{M}_{2,1}(\vec{f})(x)=\sup _{B \ni x} \sum_{k=0}^{\infty} 2^{-k n}\left(\frac{1}{|B|} \int_{B}\left|f_{1}\left(y_{1}\right)\right| \mathrm{d} y_{1}\right)\left(\frac{1}{\left|2^{k} B\right|} \int_{2^{k} B}\left|f_{2}\left(y_{2}\right)\right| \mathrm{d} y_{2}\right), \\
& \mathcal{M}_{2,2}(\vec{f})(x)=\sup _{B \ni x} \sum_{k=0}^{\infty} 2^{-k n}\left(\frac{1}{|B|} \int_{B}\left|f_{2}\left(y_{2}\right)\right| \mathrm{d} y_{2}\right)\left(\frac{1}{\left|2^{k} B\right|} \int_{2^{k} B}\left|f_{1}\left(y_{1}\right)\right| \mathrm{d} y_{1}\right),
\end{aligned}
$$

where $\vec{f}=\left(f_{1}, f_{2}\right)$ are locally integrable functions. And the following boundedness for $\mathcal{M}_{2,1}$ and $\mathcal{M}_{2,2}$ were proved in [15].

Lemma 2.2. Let $1<p_{1}, p_{2}<\infty, \frac{1}{p}=\sum_{j=1}^{2} \frac{1}{p_{j}}$, and $\vec{w}=\left(w_{1}, w_{2}\right) \in A_{\vec{p}}\left(\mathbb{R}^{2 n}\right)$. Then $\mathcal{M}_{2,1}$ and $\mathcal{M}_{2,2}$ are bounded from $L^{p_{1}}\left(w_{1}\right) \times L^{p_{2}}\left(w_{2}\right)$ to $L^{p}\left(\nu_{\vec{w}}\right)$.

Lemma 2.3. Let $b \in \mathrm{BMO}\left(\mathbb{R}^{n}\right)$. Suppose that $T$ is as in Theorem 1.5, then for any $x \in \mathbb{R}^{n}, \delta>0$ and $0<\eta<1 / 2$,

$$
\begin{aligned}
\left|\left[b, T_{\delta}\right]_{1}\left(f_{1}, f_{2}\right)(x)\right| \lesssim & M_{b}\left(f_{1}\right)(x) M\left(f_{2}\right)(x)+M_{\eta}\left(T_{b, 1}\left(f_{1}, f_{2}\right)(x)\right) \\
+ & {\left[M_{b}\left(T\left(f_{1}, f_{2}\right)^{\eta}(x)\right)\right]\left[M_{\eta}\left(T\left(f_{1}, f_{2}\right)(x)\right)\right]^{1-\eta} } \\
+ & M_{\eta}\left(T_{b, 1}\left(f_{1} \chi_{B\left(x, \delta^{1 / 2}\right)}, f_{2} \chi_{B\left(x, \delta^{1 / 2}\right)}\right)(x)\right) \\
+ & \left(\left[M_{b}\left(T\left(f_{1} \chi_{B\left(x, \delta^{1 / 2}\right)}, f_{2} \chi_{B\left(x, \delta^{1 / 2}\right)}\right)^{\eta}(x)\right)\right]\right. \\
& \left.\times\left[M_{\eta}\left(T\left(f_{1} \chi_{B\left(x, \delta^{1 / 2}\right)}, f_{2} \chi_{B\left(x, \delta^{1 / 2}\right)}\right)(x)\right)\right]^{1-\eta}\right)
\end{aligned}
$$


where $f_{1}, f_{2}$ are any bounded functions with compact support.

Proof. We adopt some ideas of [5] and [16]. Let $\delta>0$ and function $\vec{g}=\left(g_{1}, g_{2}\right)$ which is chosen later. From the size condition (1.2), we deduce that

$$
\begin{aligned}
& \left|T_{\delta}\left(g_{1}, g_{2}\right)(x)\right|=\left|\iint_{\left|x-y_{1}\right|^{2}+\left|x-y_{2}\right|^{2}>\delta} K\left(x, y_{1}, y_{2}\right) g_{1}\left(y_{1}\right) g_{2}\left(y_{2}\right) \mathrm{d} y_{1} \mathrm{~d} y_{2}\right| \\
& \leq\left|\iint_{\substack{\max \left(\left|x-y_{1}\right|^{2}+\left|x-y_{2}\right|^{2}>\delta \\
\left|y_{2}\right|\right)<\delta^{1 / 2}}} K\left(x, y_{1}, y_{2}\right) g_{1}\left(y_{1}\right) g_{2}\left(y_{2}\right) \mathrm{d} y_{1} \mathrm{~d} y_{2}\right| \\
& \quad+\left|\iint_{\max \left(\left|x-y_{1}\right|,\left|x-y_{2}\right|\right) \geq \delta^{1 / 2}} K\left(x, y_{1}, y_{2}\right) g_{1}\left(y_{1}\right) g_{2}\left(y_{2}\right) \mathrm{d} y_{1} \mathrm{~d} y_{2}\right| \\
& \lesssim M\left(g_{1}\right)(x) M\left(g_{2}\right)(x)+\left|\iint_{\max \left(\left|x-y_{1}\right|,\left|x-y_{2}\right|\right) \geq \delta^{1 / 2}} K\left(x, y_{1}, y_{2}\right) g_{1}\left(y_{1}\right) g_{2}\left(y_{2}\right) \mathrm{d} \vec{y}\right| \\
& \lesssim M\left(g_{1}\right)(x) M\left(g_{2}\right)(x)+\left|\iint_{\min \left(\left|x-y_{1}\right|,\left|x-y_{2}\right|\right) \geq \delta^{1 / 2}} K\left(x, y_{1}, y_{2}\right) g_{1}\left(y_{1}\right) g_{2}\left(y_{2}\right) \mathrm{d} \vec{y}\right| \\
& \quad+\left|\int_{\left|x-y_{1}\right|>\delta^{1 / 2}} \int_{\left|x-y_{2}\right|<\delta^{1 / 2}} K\left(x, y_{1}, y_{2}\right) g_{1}\left(y_{1}\right) g_{2}\left(y_{2}\right) \mathrm{d} y_{1} \mathrm{~d} y_{2}\right| \\
& \quad+\left|\int_{\left|x-y_{2}\right|>\delta^{1 / 2}} \int_{\left|x-y_{1}\right|<\delta^{1 / 2}} K\left(x, y_{1}, y_{2}\right) g_{1}\left(y_{1}\right) g_{2}\left(y_{2}\right) \mathrm{d} y_{1} \mathrm{~d} y_{2}\right| \\
& \lesssim M\left(g_{1}\right)(x) M\left(g_{2}\right)(x)+\left|\iint_{\min \left(\left|x-y_{1}\right|,\left|x-y_{2}\right|\right) \geq \delta^{1 / 2}} K\left(x, y_{1}, y_{2}\right) g_{1}\left(y_{1}\right) g_{2}\left(y_{2}\right) \mathrm{d} \vec{y}\right|,
\end{aligned}
$$

where the last inequality holds because

$$
\begin{aligned}
& \left|\int_{\left|x-y_{1}\right|>\delta^{1 / 2}} \int_{\left|x-y_{2}\right|<\delta^{1 / 2}} K\left(x, y_{1}, y_{2}\right) g_{1}\left(y_{1}\right) g_{2}\left(y_{2}\right) \mathrm{d} y_{1} \mathrm{~d} y_{2}\right| \\
& \lesssim \int_{\left|x-y_{1}\right|>\delta^{1 / 2}} \int_{\left|x-y_{2}\right|<\delta^{1 / 2}} \frac{\left|g_{1}\left(y_{1}\right)\right|\left|g_{2}\left(y_{2}\right)\right|}{\left(\left|x-y_{1}\right|+\left|x-y_{2}\right|\right)^{2 n}} \mathrm{~d} y_{1} \mathrm{~d} y_{2} \\
& \lesssim \int_{\left|x-y_{1}\right|>\delta^{1 / 2}} \frac{\left|g_{1}\left(y_{1}\right)\right|}{\left|x-y_{1}\right|^{n+1}} \mathrm{~d} y_{1} \int_{\left|x-y_{2}\right|<\delta^{1 / 2}} \frac{\left|g_{2}\left(y_{2}\right)\right|}{\left|x-y_{2}\right|^{n-1}} \mathrm{~d} y_{2} \\
& \lesssim M\left(g_{1}\right)(x) M\left(g_{2}\right)(x) .
\end{aligned}
$$

Denote

$$
\widetilde{T}_{\delta}\left(g_{1}, g_{2}\right)(x)=\iint_{\min \left(\left|x-y_{1}\right|,\left|x-y_{2}\right|\right) \geq \delta^{1 / 2}} K\left(x, y_{1}, y_{2}\right) g_{1}\left(y_{1}\right) g_{2}\left(y_{2}\right) \mathrm{d} y_{1} \mathrm{~d} y_{2} .
$$

Fix $\delta>0$ and let $z \in B\left(x, \frac{\delta^{1 / 2}}{8}\right)$, we obtain

$$
\begin{aligned}
& \iint_{\min \left(\left|x-y_{1}\right|,\left|x-y_{2}\right|\right) \geq \delta^{1 / 2}} K\left(z, y_{1}, y_{2}\right) g_{1}\left(y_{1}\right) g_{2}\left(y_{2}\right) \mathrm{d} y_{1} \mathrm{~d} y_{2} \\
& =T(\vec{g})(z)-T\left(g_{1} \chi_{B\left(x, \delta^{1 / 2}\right)}, g_{2} \chi_{B\left(x, \delta^{1 / 2}\right)}\right)(z)-T\left(g_{1} \chi_{B\left(x, \delta^{1 / 2}\right)}, g_{2} \chi_{B^{c}\left(x, \delta^{1 / 2}\right)}\right)(z) \\
& -T\left(g_{1} \chi_{B^{c}\left(x, \delta^{1 / 2}\right)}, g_{2} \chi_{B\left(x, \delta^{1 / 2}\right)}\right)(z) .
\end{aligned}
$$


Because $\left|z-y_{2}\right|=\left|z-x+x-y_{2}\right| \geq\left|x-y_{2}\right|-|z-x|$, we know that

$$
\begin{aligned}
& \left|T\left(g_{1} \chi_{B\left(x, \delta^{1 / 2}\right)}, g_{2} \chi_{B^{c}\left(x, \delta^{1 / 2}\right)}\right)(z)\right| \\
& \leq \int_{\left|x-y_{1}\right|<\delta^{1 / 2}} \int_{\left|x-y_{2}\right|>\delta^{1 / 2}} \frac{\left|g_{1}\left(y_{1}\right)\right|\left|g_{2}\left(y_{2}\right)\right|}{\left(\left|z-y_{1}\right|+\left|z-y_{2}\right|\right)^{2 n}} \mathrm{~d} y_{1} \mathrm{~d} y_{2} \\
& \lesssim \int_{\left|x-y_{1}\right|<\delta^{1 / 2}}\left|g_{1}\left(y_{1}\right)\right| \mathrm{d} y_{1} \sum_{k=1}^{\infty} \int_{2^{k-1} \delta^{1 / 2}<\left|x-y_{2}\right|<2^{k} \delta^{1 / 2}} \frac{\left|g_{2}\left(y_{2}\right)\right|}{\left|x-y_{2}\right|^{2 n}} \mathrm{~d} y_{2} \\
& \lesssim \sum_{k=1}^{\infty} 2^{-k n} \frac{1}{\left|B\left(x, \delta^{1 / 2}\right)\right|} \int_{B\left(x, \delta^{1 / 2}\right)}\left|g_{1}\left(y_{1}\right)\right| \mathrm{d} y_{1} \frac{1}{\left|B\left(x, 2^{k} \delta^{1 / 2}\right)\right|} \int_{B\left(x, 2^{k} \delta^{1 / 2}\right)}\left|g_{2}\left(y_{2}\right)\right| \mathrm{d} y_{2} \\
& \lesssim \mathcal{M}_{2,1}\left(g_{1}, g_{2}\right)(x),
\end{aligned}
$$

similarly, we get

$$
\left|T\left(g_{1} \chi_{B^{c}\left(x, \delta^{1 / 2}\right)}, g_{2} \chi_{B\left(x, \delta^{1 / 2}\right)}\right)(z)\right| \lesssim \mathcal{M}_{2,2}\left(g_{1}, g_{2}\right)(x) .
$$

On the other hand, by taking infimum over $\tau$ in condition (1.4), we have

$$
\left|K\left(x, y_{1}, y_{2}\right)-K\left(z, y_{1}, y_{2}\right)\right| \leq \frac{D|x-z|^{\gamma}}{\left(\left|x-y_{1}\right|+\left|x-y_{2}\right|\right)^{2 n+\gamma}},
$$

when $8|x-z|<\min _{1 \leq j \leq 2}\left|x-y_{j}\right|$. Thus,

$$
\begin{aligned}
& \left|\widetilde{T}_{\delta}\left(g_{1}, g_{2}\right)(x)-\iint_{\min \left(\left|x-y_{1}\right|,\left|x-y_{2}\right|\right) \geq \delta^{1 / 2}} K\left(z, y_{1}, y_{2}\right) g_{1}\left(y_{1}\right) g_{2}\left(y_{2}\right) \mathrm{d} y_{1} \mathrm{~d} y_{2}\right| \\
& \lesssim \iint_{\min \left(\left|x-y_{1}\right|,\left|x-y_{2}\right|\right) \geq \delta^{1 / 2}} \frac{|x-z|^{\gamma}}{\left(\left|x-y_{1}\right|+\left|x-y_{2}\right|\right)^{2 n+\gamma}}\left|g_{1}\left(y_{1}\right)\right|\left|g_{2}\left(y_{2}\right)\right| \mathrm{d} y_{1} \mathrm{~d} y_{2} \\
& \lesssim \delta^{\frac{\gamma}{2}} \iint_{\min \left(\left|x-y_{1}\right|,\left|x-y_{2}\right|\right) \geq \delta^{1 / 2}} \frac{\left|g_{1}\left(y_{1}\right)\right|\left|g_{2}\left(y_{2}\right)\right|}{\left(\left|x-y_{1}\right|+\left|x-y_{2}\right|\right)^{2 n+\gamma}} \mathrm{d} y_{1} \mathrm{~d} y_{2} \\
& \lesssim M\left(g_{1}\right)(x) M\left(g_{2}\right)(x) .
\end{aligned}
$$

In summary, for $z \in B\left(x, \frac{\delta^{1 / 2}}{8}\right)$, we obtain

$$
\begin{aligned}
\left|T_{\delta}\left(g_{1}, g_{2}\right)(x)\right| \lesssim & M\left(g_{1}\right)(x) M\left(g_{2}\right)(x)+\sum_{i=1}^{2} \mathcal{M}_{2, i}\left(g_{1}, g_{2}\right)(x) \\
& +\left|T\left(g_{1}, g_{2}\right)(z)-T\left(g_{1} \chi_{B\left(x, \delta^{1 / 2}\right)}, g_{2} \chi_{B\left(x, \delta^{1 / 2}\right)}\right)(z)\right| .
\end{aligned}
$$

For $0<\eta<\frac{1}{2}$. Raising the above inequality to the power $\eta$, integrating over $z \in B\left(x, \frac{\delta^{1 / 2}}{8}\right)$, and dividing by $\left|B\left(x, \frac{\delta^{1 / 2}}{8}\right)\right|$, we get

$$
\begin{aligned}
\left|T_{\delta}\left(g_{1}, g_{2}\right)(x)\right|^{\eta} \lesssim & {\left[\prod_{i=1}^{2} M\left(g_{i}\right)(x)\right]^{\eta}+\sum_{i=1}^{2}\left[\mathcal{M}_{2, i}\left(g_{1}, g_{2}\right)(x)\right]^{\eta} } \\
& +\frac{1}{\left|B\left(x, \frac{\delta^{1 / 2}}{8}\right)\right|} \int_{B\left(x, \frac{\delta^{1 / 2}}{8}\right)}\left|T\left(g_{1}, g_{2}\right)(z)\right|^{\eta} \mathrm{d} z \\
& +\frac{1}{\left|B\left(x, \frac{\delta^{1 / 2}}{8}\right)\right|} \int_{B\left(x, \frac{\delta^{1 / 2}}{8}\right)}\left|T\left(g_{1} \chi_{B\left(x, \delta^{1 / 2}\right)}, g_{2} \chi_{B\left(x, \delta^{1 / 2}\right)}\right)(z)\right|^{\eta} \mathrm{d} z .
\end{aligned}
$$


Let $g_{1}(\cdot)=(b(x)-b(\cdot)) f_{1}(\cdot)$ and $g_{2}(\cdot)=f_{2}(\cdot)$, we have

$$
\begin{aligned}
& \left|T_{\delta}\left((b(x)-b) f_{1}, f_{2}\right)(x)\right| \\
& \lesssim M\left((b(x)-b) f_{1}\right)(x) M\left(f_{2}\right)(x)+\sum_{i=1}^{2} \mathcal{M}_{2, i}\left((b(x)-b) f_{1}, f_{2}\right)(x) \\
& \quad+\left[\frac{1}{\left|B\left(x, \frac{\delta^{1 / 2}}{8}\right)\right|} \int_{B\left(x, \frac{\delta^{1 / 2}}{8}\right)}\left|T\left((b(x)-b) f_{1}, f_{2}\right)(z)\right|^{\eta} \mathrm{d} z\right]^{\frac{1}{\eta}} \\
& +\left[\frac{1}{\left|B\left(x, \frac{\delta^{1 / 2}}{8}\right)\right|} \int_{B\left(x, \frac{\delta^{1 / 2}}{8}\right)}\left|T\left((b(x)-b) f_{1} \chi_{B\left(x, \delta^{1 / 2}\right)}, f_{2} \chi_{B\left(x, \delta^{1 / 2}\right)}\right)(z)\right|^{\eta} \mathrm{d} z\right]^{\frac{1}{\eta}} \\
& \lesssim \\
& \quad M_{b}\left(f_{1}\right)(x) M\left(f_{2}\right)(x)+\sum_{i=1}^{2} \mathcal{M}_{2, i}\left((b(x)-b) f_{1}, f_{2}\right)(x) \\
& +\left[\frac{1}{\left|B\left(x, \frac{\delta^{1 / 2}}{8}\right)\right|} \int_{B\left(x, \frac{\delta^{1 / 2}}{8}\right)}\left|T\left((b(x)-b(z)+b(z)-b) f_{1}, f_{2}\right)(z)\right|^{\eta} \mathrm{d} z\right]^{\frac{1}{\eta}} \\
& \quad+\left[\frac{1}{\left|B\left(x, \frac{\delta^{1 / 2}}{8}\right)\right|} \int_{B\left(x, \frac{\delta^{1 / 2}}{8}\right)} \mid T((b(x)-b(z)+b(z)-b)\right. \\
& \left.\left.\quad \times f_{1} \chi_{B\left(x, \delta^{1 / 2}\right)}, f_{2} \chi_{B\left(x, \delta^{1 / 2}\right)}\right)\left.(z)\right|^{\eta} \mathrm{d} z\right]^{\frac{1}{\eta}} \cdot
\end{aligned}
$$

Hence, by Hölder's inequality and the fact that

$$
\sum_{i=1}^{2} \mathcal{M}_{2, i}\left((b(x)-b) f_{1}, f_{2}\right)(x) \lesssim M_{b}\left(f_{1}\right)(x) M\left(f_{2}\right)(x),
$$

we know that

$$
\begin{aligned}
\left|\left[b, T_{\delta}\right]_{1}\left(f_{1}, f_{2}\right)(x)\right| \lesssim & M_{b}\left(f_{1}\right)(x) M\left(f_{2}\right)(x)+M_{\eta}\left(T_{b, 1}\left(f_{1}, f_{2}\right)(x)\right) \\
+ & {\left[M_{b}\left(T\left(f_{1}, f_{2}\right)^{\eta}(x)\right)\right]\left[M_{\eta}\left(T\left(f_{1}, f_{2}\right)(x)\right)\right]^{1-\eta} } \\
+ & M_{\eta}\left(T_{b, 1}\left(f_{1} \chi_{B\left(x, \delta^{1 / 2}\right)}, f_{2} \chi_{B\left(x, \delta^{1 / 2}\right)}\right)(x)\right) \\
+ & \left(\left[M_{b}\left(T\left(f_{1} \chi_{B\left(x, \delta^{1 / 2}\right)}, f_{2} \chi_{B\left(x, \delta^{1 / 2}\right)}\right)^{\eta}(x)\right)\right]\right. \\
& \left.\times\left[M_{\eta}\left(T\left(f_{1} \chi_{B\left(x, \delta^{1 / 2}\right)}, f_{2} \chi_{B\left(x, \delta^{1 / 2}\right)}\right)(x)\right)\right]^{1-\eta}\right) .
\end{aligned}
$$

Remark 2.4. Let $b \in \mathrm{BMO}\left(\mathbb{R}^{n}\right), T$ is as in Theorem 1.5. Then for any $x \in \mathbb{R}^{n}, \delta>$ 0 and $0<\eta<1 / 2,\left[b, T_{\delta}\right]_{2}\left(f_{1}, f_{2}\right)(x)$ has the similar estimate as $\left[b, T_{\delta}\right]_{1}\left(f_{1}, f_{2}\right)(x)$ in Lemma 2.3.

Lemma 2.5. Let $0<\lambda, \lambda_{1}, \lambda_{2}<n, p_{1}, p_{2} \in(1, \infty), p \in(1, \infty)$ such that $1 / p=$ $1 / p_{1}+1 / p_{2}$ and $\lambda / p=\lambda_{1} / p_{1}+\lambda_{2} / p_{2}$. Suppose that $T$ is bounded from $L^{p_{1}}\left(w_{1}\right) \times$ $L^{p_{2}}\left(w_{2}\right)$ to $L^{p}\left(\nu_{\vec{w}}\right)$ for $\vec{w}=\left(w_{1}, w_{2}\right) \in A_{1}\left(\mathbb{R}^{n}\right) \times A_{1}\left(\mathbb{R}^{n}\right)$ and $\nu_{\vec{w}} \in A_{1}\left(\mathbb{R}^{n}\right)$. Then $T$ is bounded from $L^{p_{1}, \lambda_{1}}\left(\mathbb{R}^{n}\right) \times L^{p_{2}, \lambda_{2}}\left(\mathbb{R}^{n}\right)$ to $L^{p, \lambda}\left(\mathbb{R}^{n}\right)$. 
Proof. For any fixed ball $B=B(s, r)$ we decompose $f_{1}, f_{2}$ as $f_{1}=f_{1}^{0}+\sum_{k=1}^{\infty} f_{1}^{k}=$ $f_{1} \chi_{B}+\sum_{k=1}^{\infty} f_{1}^{k} \chi_{2^{k} B \backslash 2^{k-1} B}, f_{2}=f_{2}^{0}+\sum_{j=1}^{\infty} f_{2}^{j}=f_{2} \chi_{B}+\sum_{j=1}^{\infty} f_{2}^{j} \chi_{2^{j} B \backslash 2^{j-1} B}$. Hence,

$$
\begin{aligned}
& \left(\frac{1}{r^{\lambda}} \int_{B}\left|T\left(f_{1}^{0}, f_{2}^{0}\right)(x)\right|^{p} \mathrm{~d} x\right)^{\frac{1}{p}} \\
& \lesssim \frac{1}{r^{\lambda / p}}\left(\int_{B}\left|T\left(f_{1}^{0}, f_{2}^{0}\right)(x)\right|^{p} \mathrm{~d} x\right)^{\frac{1}{p}}
\end{aligned}
$$

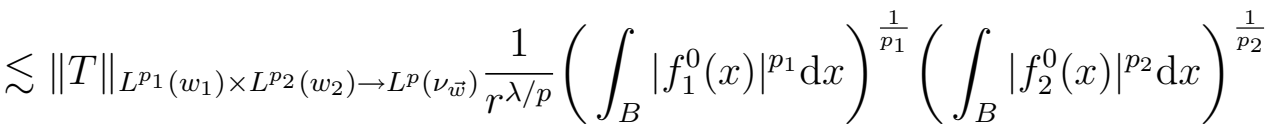

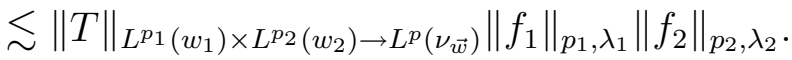

Let $\max \left(\frac{\lambda_{1}}{n}, \frac{\lambda_{2}}{n}\right)<\theta<1$, then $\left(M \chi_{B}(x)\right)^{\theta} \in A_{1}\left(\mathbb{R}^{n}\right)$ (see [8]). By the fact that $M \chi_{B}(x) \lesssim 2^{-j n}$ when $x \in 2^{j+1} B \backslash 2^{j} B$, we have

$$
\begin{aligned}
& \sum_{j=1}^{\infty}\left(\frac{1}{r^{\lambda}} \int_{B}\left|T\left(f_{1}^{0}, f_{2}^{j}\right)(x)\right|^{p} \mathrm{~d} x\right)^{\frac{1}{p}} \lesssim \sum_{j=1}^{\infty} \frac{1}{r^{\lambda / p}}\left(\int_{\mathbb{R}^{n}}\left|T\left(f_{1}^{0}, f_{2}^{j}\right)(x)\right|^{p}\left(\chi_{B}(x)\right)^{\theta} \mathrm{d} x\right)^{\frac{1}{p}} \\
& \lesssim \sum_{j=1}^{\infty} \frac{1}{r^{\lambda / p}}\left(\int_{\mathbb{R}^{n}}\left|T\left(f_{1}^{0}, f_{2}^{j}\right)(x)\right|^{p}\left(M \chi_{B}(x)\right)^{\theta} \mathrm{d} x\right)^{\frac{1}{p}} \\
& \lesssim\|T\|_{L^{p_{1}}\left(w_{1}\right) \times L^{p_{2}\left(w_{2}\right) \rightarrow L^{p}\left(\nu_{\vec{w}}\right)}} \sum_{j=1}^{\infty} \frac{1}{r^{\lambda / p}}\left(\int_{B}\left|f_{1}(x)\right|^{p_{1}}\left(M \chi_{B}(x)\right)^{\theta} \mathrm{d} x\right)^{\frac{1}{p_{1}}} \\
& \times\left(\int_{2^{j} B \backslash 2^{j-1} B}\left|f_{2}(x)\right|^{p_{2}}\left(M \chi_{B}(x)\right)^{\theta} \mathrm{d} x\right)^{\frac{1}{p_{2}}} \\
& \lesssim\|T\|_{L^{p_{1}}\left(w_{1}\right) \times L^{p_{2}}\left(w_{2}\right) \rightarrow L^{p}\left(\nu_{\vec{w}}\right)} \sum_{j=1}^{\infty} \frac{1}{r^{\lambda / p}}\left(\int_{B}\left|f_{1}(x)\right|^{p_{1}} \mathrm{~d} x\right)^{\frac{1}{p_{1}}} \\
& \times 2^{\frac{-j n \theta}{p_{2}}}\left(\int_{2^{j} B}\left|f_{2}(x)\right|^{p_{2}} \mathrm{~d} x\right)^{\frac{1}{p_{2}}} \\
& \lesssim\|T\|_{L^{p_{1}}\left(w_{1}\right) \times L^{p_{2}\left(w_{2}\right) \rightarrow L^{p}\left(\nu_{\vec{w}}\right)}} \sum_{j=1}^{\infty} 2^{-\frac{j}{p_{2}}\left(n \theta-\lambda_{2}\right)}\left\|f_{1}\right\|_{p_{1}, \lambda_{1}}\left\|f_{2}\right\|_{p_{2}, \lambda_{2}} \\
& \lesssim\|T\|_{L^{p_{1}}\left(w_{1}\right) \times L^{p_{2}}\left(w_{2}\right) \rightarrow L^{p}\left(\nu_{\vec{w}}\right)}\left\|f_{1}\right\|_{p_{1}, \lambda_{1}}\left\|f_{2}\right\|_{p_{2}, \lambda_{2}},
\end{aligned}
$$

similarly, we get

$$
\sum_{k=1}^{\infty}\left(\frac{1}{r^{\lambda}} \int_{B}\left|T\left(f_{1}^{k}, f_{2}^{0}\right)(x)\right|^{p} \mathrm{~d} x\right)^{\frac{1}{p}} \lesssim\|T\|_{L^{p_{1}\left(w_{1}\right) \times L^{p_{2}}\left(w_{2}\right) \rightarrow L^{p}\left(\nu_{\vec{w}}\right)} \|}\left\|f_{1}\right\|_{p_{1}, \lambda_{1}}\left\|f_{2}\right\|_{p_{2}, \lambda_{2}},
$$

and

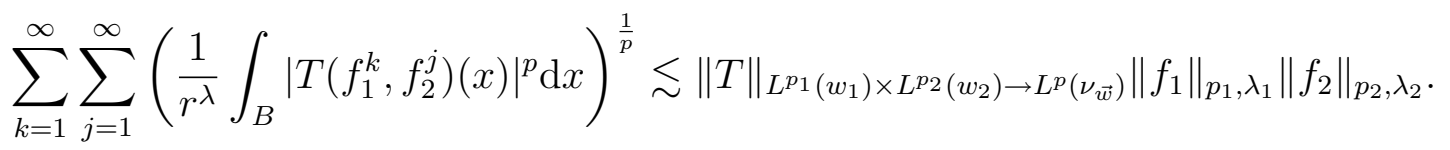


In summary, we obtain

$$
\begin{aligned}
& \left\|T\left(f_{1}, f_{2}\right)\right\|_{p, \lambda} \lesssim\left\|T\left(f_{1}^{0}, f_{2}^{0}\right)\right\|_{p, \lambda}+\sum_{j=1}^{\infty}\left\|T\left(f_{1}^{0}, f_{2}^{j}\right)\right\|_{p, \lambda} \\
& +\sum_{k=1}^{\infty}\left\|T\left(f_{1}^{k}, f_{2}^{0}\right)\right\|_{p, \lambda}+\sum_{j=1}^{\infty} \sum_{k=1}^{\infty}\left\|T\left(f_{1}^{k}, f_{2}^{j}\right)\right\|_{p, \lambda} \\
& \lesssim\|T\|_{L^{p_{1}}\left(w_{1}\right) \times L^{p_{2}\left(w_{2}\right) \rightarrow L^{p}\left(\nu_{\vec{w}}\right)}}\left\|f_{1}\right\|_{p_{1}, \lambda_{1}}\left\|f_{2}\right\|_{p_{2}, \lambda_{2}} .
\end{aligned}
$$

Remark 2.6. Lemma 2.5 is also true for the linear case.

Now, we are ready to prove Theorem 1.7.

Proof. We only write out the proof of $T_{b, 1}^{*}(\vec{f})(x)$, the other can be obtained by symmetry. From Lemma 2.3, we deduce that

$$
\begin{aligned}
\left\|T_{b, 1}^{*}(\vec{f})\right\|_{p, \lambda} \lesssim & \left\|M_{b}\left(f_{1}\right)(x) M\left(f_{2}\right)(x)\right\|_{p, \lambda}+\left\|M_{\eta}\left(T_{b, 1}\left(f_{1}, f_{2}\right)(x)\right)\right\|_{p, \lambda} \\
+ & \left\|\left[M_{b}\left(T\left(f_{1}, f_{2}\right)^{\eta}(x)\right)\right]\left[M_{\eta}\left(T\left(f_{1}, f_{2}\right)(x)\right)\right]^{1-\eta}\right\|_{p, \lambda} \\
+ & \sup _{\delta>0}\left\|M_{\eta}\left(T_{b, 1}\left(f_{1} \chi_{B\left(x, \delta^{1 / 2}\right)}, f_{2} \chi_{B\left(x, \delta^{1 / 2}\right)}\right)(x)\right)\right\|_{p, \lambda} \\
+ & \sup _{\delta>0} \|\left(\left[M_{b}\left(T\left(f_{1} \chi_{B\left(x, \delta^{1 / 2}\right)}, f_{2} \chi_{B\left(x, \delta^{1 / 2}\right)}\right)^{\eta}(x)\right)\right]\right. \\
& \left.\times\left[M_{\eta}\left(T\left(f_{1} \chi_{B\left(x, \delta^{1 / 2}\right)}, f_{2} \chi_{B\left(x, \delta^{1 / 2}\right)}\right)(x)\right)\right]^{1-\eta}\right) \|_{p, \lambda} .
\end{aligned}
$$

By Hölder's inequality, Lemma 2.1, Lemma 2.5 and (2.1), we know that, there exists a $\eta_{0}>1$ such that

$$
\begin{aligned}
& \left\|M_{b}\left(f_{1}\right)(x) M\left(f_{2}\right)(x)\right\|_{p, \lambda} \\
& \lesssim\left\|M_{b}\left(f_{1}\right)(x)\right\|_{p_{1}, \lambda_{1}}\left\|M\left(f_{2}\right)(x)\right\|_{p_{2}, \lambda_{2}} \\
& \lesssim\left\|M_{b}\right\|_{L^{p_{1}}(w) \rightarrow L^{p_{1}}(w)}\left\|f_{1}\right\|_{p_{1}, \lambda_{1}}\left\|f_{2}\right\|_{p_{2}, \lambda_{2}} \\
& \lesssim\|b\|_{\mathrm{BMO}\left(\mathbb{R}^{n}\right)}\left\|M_{\eta_{0}}\right\|_{L^{p_{1}}(w) \rightarrow L^{p_{1}}(w)}\left\|f_{1}\right\|_{p_{1}, \lambda_{1}}\left\|f_{2}\right\|_{p_{2}, \lambda_{2}} \\
& \lesssim\|b\|_{\mathrm{BMO}\left(\mathbb{R}^{n}\right)}\left\|f_{1}\right\|_{p_{1}, \lambda_{1}}\left\|f_{2}\right\|_{p_{2}, \lambda_{2}} .
\end{aligned}
$$

where $w \in A_{1}\left(\mathbb{R}^{n}\right)$. From [3] and Lemma 2.5, we get

$$
\begin{aligned}
\left\|M_{\eta}\left(T_{b, 1}\left(f_{1}, f_{2}\right)(x)\right)\right\|_{p, \lambda} & \lesssim\left\|T_{b, 1}\left(f_{1}, f_{2}\right)(x)\right\|_{p, \lambda} \\
& \lesssim\|b\|_{\mathrm{BMO}\left(\mathbb{R}^{n}\right)}\left\|f_{1}\right\|_{p_{1}, \lambda_{1}}\left\|f_{2}\right\|_{p_{2}, \lambda_{2}} .
\end{aligned}
$$

By Hölder's inequality, Lemma 2.3 and Lemma 2.5, we have

$$
\begin{aligned}
& \left\|\left[M_{b}\left(T\left(f_{1}, f_{2}\right)^{\eta}(x)\right)\right]\left[M_{\eta}\left(T\left(f_{1}, f_{2}\right)(x)\right)\right]^{1-\eta}\right\|_{p, \lambda} \\
& \lesssim\left\|\left[M_{b}\left(T\left(f_{1}, f_{2}\right)^{\eta}(x)\right)\right]\right\|_{p / \eta, \lambda}\left\|\left[M_{\eta}\left(T\left(f_{1}, f_{2}\right)(x)\right)\right]^{1-\eta}\right\|_{p /(1-\eta), \lambda} \\
& \lesssim\|b\|_{\operatorname{BMO}\left(\mathbb{R}^{n}\right)}\left\|T\left(f_{1}, f_{2}\right)^{\eta}(x)\right\|_{p / \eta, \lambda}\left\|M_{\eta}\left(T\left(f_{1}, f_{2}\right)(x)\right)\right\|_{p, \lambda}^{1-\eta} \\
& \lesssim\|b\|_{\operatorname{BMO}\left(\mathbb{R}^{n}\right)}\left\|f_{1}\right\|_{p_{1}, \lambda_{1}}^{\eta}\left\|f_{2}\right\|_{p_{2}, \lambda_{2}}^{\eta}\left\|f_{1}\right\|_{p_{1}, \lambda_{1}}^{1-\eta}\left\|f_{2}\right\|_{p_{2}, \lambda_{2}}^{1-\eta} \\
& \lesssim\|b\|_{\mathrm{BMO}\left(\mathbb{R}^{n}\right)}\left\|f_{1}\right\|_{p_{1}, \lambda_{1}}\left\|f_{2}\right\|_{p_{2}, \lambda_{2}} .
\end{aligned}
$$


Similarly, we obtain

$$
\begin{aligned}
& \sup _{\delta>0}\left\|M_{\eta}\left(T_{b, 1}\left(f_{1} \chi_{B\left(x, \delta^{1 / 2}\right)}, f_{2} \chi_{B\left(x, \delta^{1 / 2}\right)}\right)(x)\right)\right\|_{p, \lambda} \lesssim\|b\|_{\mathrm{BMO}\left(\mathbb{R}^{n}\right)}\left\|f_{1}\right\|_{p_{1}, \lambda_{1}}\left\|f_{2}\right\|_{p_{2}, \lambda_{2}}, \\
& \sup _{\delta>0} \|\left(\left[M_{b}\left(T\left(f_{1} \chi_{B\left(x, \delta^{1 / 2}\right)}, f_{2} \chi_{B\left(x, \delta^{1 / 2}\right)}\right)^{\eta}(x)\right)\right]\right. \\
& \left.\quad \times\left[M_{\eta}\left(T\left(f_{1} \chi_{B\left(x, \delta^{1 / 2}\right)}, f_{2} \chi_{B\left(x, \delta^{1 / 2}\right)}\right)(x)\right)\right]^{1-\eta}\right) \|_{p, \lambda} \\
& \quad \lesssim\|b\|_{\mathrm{BMO}\left(\mathbb{R}^{n}\right)}\left\|f_{1}\right\|_{p_{1}, \lambda_{1}}\left\|f_{2}\right\|_{p_{2}, \lambda_{2}} .
\end{aligned}
$$

\section{Proof of Theorem 1.5}

Lemma 3.1. Let $0<\lambda, \lambda_{1}, \lambda_{2}<n, p_{1}, p_{2} \in(1, \infty), p \in(1, \infty)$ such that $1 / p=$ $1 / p_{1}+1 / p_{2}$ and $\lambda / p=\lambda_{1} / p_{1}+\lambda_{2} / p_{2}$. Suppose that $T$ is as in Theorem 1.5, then for all $0<\eta<1 / 2, x \in \mathbb{R}^{n}$ and all $\vec{f}$ in the product of $L^{p_{j}, \lambda_{j}}\left(\mathbb{R}^{n}\right)$,

$$
T^{*}(\vec{f})(x) \lesssim\left(M_{\eta}(T(\vec{f}))(x)\right)+\sum_{i=1}^{2} \mathcal{M}_{2, i}(\vec{f})(x)+M\left(f_{1}\right)(x) M\left(f_{2}\right)(x) .
$$

The proof of the Lemma 3.1 is similar to that of [16, Theorem 1] and Lemma 2.3, so we leave it to the interested reader. From Lemma 2.2, Lemma 2.5, Lemma 3.1 and [3], it is easy to find that

$$
\left\|T^{*}(\vec{f})(x)\right\|_{p, \lambda} \lesssim\left\|f_{1}\right\|_{p_{1}, \lambda_{1}}\left\|f_{2}\right\|_{p_{2}, \lambda_{2}}
$$

for $\lambda, \lambda_{1}, \lambda_{2}, p, p_{1}, p_{2}$ in Lemma 3.1 .

Lemma 3.2. Let $1 \leq p<\infty, 0<\lambda<n$ and $\mathcal{H} \subset L^{p, \lambda}\left(\mathbb{R}^{n}\right)$, if

(1) $\sup _{f \in \mathcal{H}}\|f\|_{p, \lambda}<\infty$;

(2) $\lim _{A \rightarrow \infty}\left\|f \chi_{\{|x|>A\}}\right\|_{p, \lambda}=0$ uniformly for $f \in \mathcal{H}$;

(3) $\lim _{t \rightarrow 0}\|f(\cdot+t)-f(\cdot)\|_{p, \lambda}=0$ uniformly for $f \in \mathcal{H}$.

Then $\mathcal{H}$ is strongly precompact set in $L^{p, \lambda}\left(\mathbb{R}^{n}\right)$.

This Lemma was given in [6].

Now, we are ready to prove Theorem 1.5.

Proof. We will work with the commutator $T_{b, 1}^{*}$ first, and the proof of commutator $T_{b, 2}^{*}$ can be got by symmetry. By Theorem 1.7, it suffices to show the result for $b \in C_{c}^{\infty}\left(\mathbb{R}^{n}\right)$. Suppose $f_{1}, f_{2}$ belong to

$$
B_{1}\left(L^{p_{1}, \lambda_{1}}\right) \times B_{1}\left(L^{p_{2}, \lambda_{2}}\right)=\left\{\left(f_{1}, f_{2}\right):\left\|f_{1}\right\|_{p_{1}, \lambda_{1}},\left\|f_{2}\right\|_{p_{2}, \lambda_{2}} \leq 1\right\} .
$$

We need to prove the following three conditions hold:

(a) $T_{b, 1}^{*}\left(B_{1}\left(L^{p_{1}, \lambda_{1}}\right) \times B_{1}\left(L^{p_{2}, \lambda_{2}}\right)\right)$ is bounded in $L^{p, \lambda}\left(\mathbb{R}^{n}\right)$;

(b) For every $s \in \mathbb{R}^{n}$ and $r>0, \lim _{A \rightarrow \infty} \frac{1}{r^{\lambda}} \int_{B(s, r)}\left|T_{b, 1}^{*}\left(f_{1}, f_{2}\right)(x) \chi_{\{|x|>A\}}(x)\right|^{p} \mathrm{~d} x=$ 0 
(c) Given $0<\xi<1 / 8$, there exists a sufficiently small $t_{0}\left(t_{0}<\xi^{2}\right)$ such that for all $0<|t|<t_{0}$, we have

$$
\left\|T_{b, 1}^{*}\left(f_{1}, f_{2}\right)(\cdot)-T_{b, 1}^{*}\left(f_{1}, f_{2}\right)(\cdot+t)\right\|_{p, \lambda} \leq C \xi .
$$

It is easy to find that the condition (a) holds because of the boundedness of $T_{b, 1}^{*}$ in Theorem 1.7. Now, we prove the condition (b) first. We pick $A>1$ sufficiently large so that $|x|>A$ implies $x \notin \operatorname{supp} b$. In particular, let $R>0$ be large enough such that $\operatorname{supp} b \subset B(0, R), A \gg \max (2 R, 1)$ and $1<p_{0}<\min \left\{p_{1}, p_{2}\right\}$,

$$
\begin{aligned}
& \left|T_{b, 1}^{*}\left(f_{1}, f_{2}\right)(x)\right| \\
& =\sup _{\delta>0}\left|\iint_{\left|x-y_{1}\right|^{2}+\left|x-y_{2}\right|^{2}>\delta} K\left(x, y_{1}, y_{2}\right)\left(b\left(y_{1}\right)-b(x)\right) f_{1}\left(y_{1}\right) f_{2}\left(y_{2}\right) \mathrm{d} \vec{y}\right| \\
& \lesssim\|b\|_{L^{\infty}} \int_{\left|y_{1}\right| \leq R} \int_{\mathbb{R}^{n}} \frac{\left|f_{1}\left(y_{1}\right)\right|\left|f_{2}\left(y_{2}\right)\right|}{\left(\left|x-y_{1}\right|+\left|x-y_{2}\right|\right)^{2 n}} \mathrm{~d} y_{1} \mathrm{~d} y_{2} \\
& \lesssim \int_{\left|x-y_{1}\right| \leq R} \int_{\mathbb{R}^{n}} \frac{\left|f_{1}\left(x-y_{1}\right)\right|\left|f_{2}\left(x-y_{2}\right)\right|}{\left(\left|y_{1}\right|+\left|y_{2}\right|\right)^{2 n}} \mathrm{~d} y_{1} \mathrm{~d} y_{2} \\
& \lesssim \int_{\left|x-y_{1}\right| \leq R} \frac{\left|f_{1}\left(x-y_{1}\right)\right|}{\left|y_{1}\right|^{n / p_{0}}} \mathrm{~d} y_{1} \int_{\mathbb{R}^{n}} \frac{\left|f_{2}\left(x-y_{2}\right)\right|}{\left(1+\left|y_{2}\right|\right)^{n+n / p_{0}^{\prime}}} \mathrm{d} y_{2} \\
& \lesssim R^{n / p_{1}^{\prime}}\left(\int_{\left|x-y_{1}\right| \leq R} \frac{\left|f_{1}\left(x-y_{1}\right)\right|^{p_{1}}}{\left|y_{1}\right|^{n p_{1} / p_{0}}} \mathrm{~d} y_{1}\right)^{1 / p_{1}}\left(\int_{\mathbb{R}^{n}} \frac{\left|f_{2}\left(x-y_{2}\right)\right|}{\left(1+\left|y_{2}\right|\right)^{n+n / p_{0}^{\prime}}} \mathrm{d} y_{2}\right) .
\end{aligned}
$$

Hence, for every $s \in \mathbb{R}^{n}$ and $r>0$, by Hölder's inequality and Minkowski's inequality

$$
\begin{aligned}
& \left(\frac{1}{r^{\lambda}} \int_{B(s, r)}\left|T_{b, 1}^{*}\left(f_{1}, f_{2}\right)(x)\right|^{p} \chi_{\{|x|>A\}}(x) \mathrm{d} x\right)^{1 / p} \\
& \lesssim R^{n / p_{1}^{\prime}}\left(\frac{1}{r^{\lambda}} \int_{B(s, r)}\left(\int_{\left|x-y_{1}\right| \leq R} \frac{\left|f_{1}\left(x-y_{1}\right)\right|^{p_{1}} \chi_{\{|x|>A\}}(x)}{\left|y_{1}\right|^{n p_{1} / p_{0}}} \mathrm{~d} y_{1}\right)^{p / p_{1}}\right. \\
& \left.\times\left(\int_{\mathbb{R}^{n}} \frac{\left|f_{2}\left(x-y_{2}\right)\right|}{\left(1+\left|y_{2}\right|\right)^{n+n / p_{0}^{\prime}}} \mathrm{d} y_{2}\right)^{p} \mathrm{~d} x\right)^{1 / p} \\
& \lesssim R^{n / p_{1}^{\prime}}\left(\frac{1}{r^{\lambda_{1}}} \int_{B(s, r)} \int_{\left|x-y_{1}\right| \leq R} \frac{\left|f_{1}\left(x-y_{1}\right)\right|^{p_{1}} \chi_{\{|x|>A\}}(x)}{\left|y_{1}\right|^{n p_{1} / p_{0}}} \mathrm{~d} y_{1} \mathrm{~d} x\right)^{1 / p_{1}} \\
& \times\left(\frac{1}{r^{\lambda_{2}}} \int_{B(s, r)}\left(\int_{\mathbb{R}^{n}} \frac{\left|f_{2}\left(x-y_{2}\right)\right|}{\left(1+\left|y_{2}\right|\right)^{n+n / p_{0}^{\prime}}} \mathrm{d} y_{2}\right)^{p_{2}} \mathrm{~d} x\right)^{1 / p_{2}} \\
& \lesssim R^{n / p_{1}^{\prime}}\left(\int_{\left|x-y_{1}\right| \leq R} \frac{1}{r^{\lambda_{1}}} \int_{B(s, r)}\left|f_{1}\left(x-y_{1}\right)\right|^{p_{1}} \chi_{\{|x|>A\}}(x) \mathrm{d} x \frac{1}{\left|y_{1}\right|^{n p_{1} / p_{0}}} \mathrm{~d} y_{1}\right)^{1 / p_{1}} \\
& \times\left(\int_{\mathbb{R}^{n}}\left(\frac{1}{r^{\lambda_{2}}} \int_{B(s, r)}\left|f_{2}\left(x-y_{2}\right)\right|^{p_{2}} \mathrm{~d} x\right)^{1 / p_{2}} \frac{1}{\left(1+\left|y_{2}\right|\right)^{n+n / p_{0}^{\prime}}} \mathrm{d} y_{2}\right)
\end{aligned}
$$




$$
\begin{aligned}
\lesssim & R^{n / p_{1}^{\prime}}\left(\int_{\left|y_{1}\right| \geq A-R} \frac{1}{\left|y_{1}\right|^{n p_{1} / p_{0}}} \mathrm{~d} y_{1}\right)^{1 / p_{1}}\left(\int_{\mathbb{R}^{n}} \frac{1}{\left(1+\left|y_{2}\right|\right)^{n+n / p_{0}^{\prime}}} \mathrm{d} y_{2}\right) \\
& \times\left\|f_{1}\right\|_{p_{1}, \lambda_{1}}\left\|f_{2}\right\|_{p_{2}, \lambda_{2}} \\
\lesssim & R^{n / p_{1}^{\prime}}(A-R)^{n\left(\frac{1}{p_{1}}-\frac{1}{p_{0}}\right)} \rightarrow 0,
\end{aligned}
$$

when $A \rightarrow \infty$.

So, it suffices to verify condition (c). Denote

$$
K^{\delta}\left(x, y_{1}, y_{2}\right)=K\left(x, y_{1}, y_{2}\right) \chi_{\left|x-y_{1}\right|^{2}+\left|x-y_{2}\right|^{2}>\delta}
$$

To prove (3.2), we need to decompose the expression inside the $L^{p, \lambda}\left(\mathbb{R}^{n}\right)$ norm as follows

$$
\begin{aligned}
& T_{b, 1}^{*}\left(f_{1}, f_{2}\right)(x)-T_{b, 1}^{*}\left(f_{1}, f_{2}\right)(x+t) \\
& \lesssim \sup _{\delta>0}\left|\iint_{\min \left(\left|x-y_{1}\right|,\left|x-y_{2}\right|\right)>\frac{|t|}{\xi}} K^{\delta}\left(x, y_{1}, y_{2}\right)(b(x+t)-b(x)) \prod_{j=1}^{2} f_{j}\left(y_{j}\right) \mathrm{d} \vec{y}\right| \\
& +\sup _{\delta>0} \mid \iint_{\min \left(\left|x-y_{1}\right|,\left|x-y_{2}\right|\right)>\frac{|t|}{\xi}}\left(K^{\delta}\left(x, y_{1}, y_{2}\right)-K^{\delta}\left(x+t, y_{1}, y_{2}\right)\right) \\
& \times\left(b\left(y_{1}\right)-b(x+t)\right) \prod_{j=1}^{2} f_{j}\left(y_{j}\right) \mathrm{d} \vec{y} \mid \\
& +\sup _{\delta>0}\left|\iint_{\min \left(\left|x-y_{1}\right|,\left|x-y_{2}\right|\right)<\frac{|t|}{\xi}} K^{\delta}\left(x, y_{1}, y_{2}\right)\left(b\left(y_{1}\right)-b(x)\right) \prod_{j=1}^{2} f_{j}\left(y_{j}\right) \mathrm{d} \vec{y}\right| \\
& +\sup _{\delta>0}\left|\iint_{\min \left(\left|x-y_{1}\right|,\left|x-y_{2}\right|\right)<\frac{|t|}{\xi}} K^{\delta}\left(x+t, y_{1}, y_{2}\right)\left(b(x+t)-b\left(y_{1}\right)\right) \prod_{j=1}^{2} f_{j}\left(y_{j}\right) \mathrm{d} \vec{y}\right| \\
& =I_{1}+I_{2}+I_{3}+I_{4} \text {. }
\end{aligned}
$$

It is easy to see that

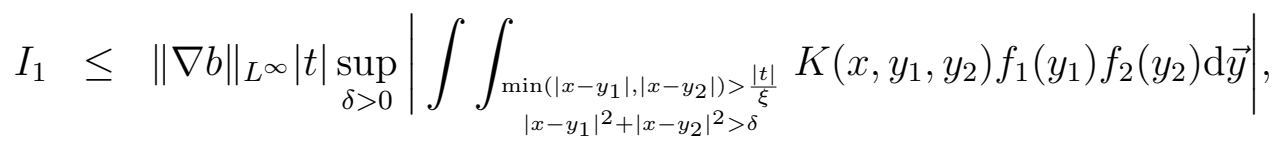

and

$$
\begin{aligned}
& \mid \iint_{\left|x-y_{1}\right|^{2}+\left|x-y_{2}\right|^{2}>\delta} K\left(x, y_{1}, y_{2}\right) f_{1}\left(y_{1}\right) f_{2}\left(y_{2}\right) \mathrm{d} y_{1} \mathrm{~d} y_{2} \\
& -\iint_{\substack{\min \left(\left|x-y_{1}\right|,\left|x-y_{2}\right|\right)>\frac{|t|}{\xi} \\
\left|x-y_{1}\right|^{2}+\left|x-y_{2}\right|^{2}>\delta}} K\left(x, y_{1}, y_{2}\right) f_{1}\left(y_{1}\right) f_{2}\left(y_{2}\right) \mathrm{d} y_{1} \mathrm{~d} y_{2} \mid \\
& =\left|\iint_{\substack{\min \left(\left|x-y_{1}\right|,\left|x-y_{2}\right|\right)<\frac{|t|}{\left|x-y_{1}\right|^{2}+\left|x-y_{2}\right|^{2}>\delta} \\
\mid x-y_{1}}} K\left(x, y_{1}, y_{2}\right) f_{1}\left(y_{1}\right) f_{2}\left(y_{2}\right) \mathrm{d} y_{1} \mathrm{~d} y_{2}\right|
\end{aligned}
$$




$$
\begin{aligned}
& \leq \iint_{\substack{\min \left(\left|x-y_{1}\right|,\left|x-y_{2}\right|\right)<\frac{|t|}{\zeta}\left|x-y_{1}\right|^{2}+\left|x-y_{2}\right|^{2}>\delta \\
\mid f_{1}}} \frac{\left|f_{1}\left(y_{1}\right)\right|\left|f_{2}\left(y_{2}\right)\right|}{\left(\left|x-y_{1}\right|+\left|x-y_{2}\right|\right)^{2 n}} \mathrm{~d} y_{1} \mathrm{~d} y_{2} \\
& \leq \int_{\left|x-y_{1}\right|<\frac{|t|}{\xi}} \int_{\left|x-y_{2}\right|>\frac{|t|}{\xi}} \frac{\left|f_{1}\left(y_{1}\right)\right|\left|f_{2}\left(y_{2}\right)\right|}{\left(\left|x-y_{1}\right|+\left|x-y_{2}\right|\right)^{2 n}} \mathrm{~d} y_{1} \mathrm{~d} y_{2} \\
& +\int_{\left|x-y_{1}\right|>\frac{|t|}{\xi}} \int_{\left|x-y_{2}\right|<\mid \frac{|t|}{\xi}} \frac{\left|f_{1}\left(y_{1}\right)\right|\left|f_{2}\left(y_{2}\right)\right|}{\left(\left|x-y_{1}\right|+\left|x-y_{2}\right|\right)^{2 n}} \mathrm{~d} y_{1} \mathrm{~d} y_{2} \\
& \lesssim \int_{\left|y_{1}\right|<\frac{|t|}{\xi}} \int_{\left|y_{2}\right|>\frac{|t|}{\xi}} \frac{\left|f_{1}\left(x-y_{1}\right)\right|\left|f_{2}\left(x-y_{2}\right)\right|}{\left(\left|y_{1}\right|+\left|y_{2}\right|\right)^{2 n}} \mathrm{~d} y_{1} \mathrm{~d} y_{2},
\end{aligned}
$$

provided $(\delta / 2)^{1 / 2}>|t| / \xi$. Then for every $s \in \mathbb{R}^{n}$ and $r>0$, by Hölder's inequality, Minkowski's inequality and (3.1), we have

$$
\begin{aligned}
& \left(\frac{1}{r^{\lambda}} \int_{B(s, r)}\left|I_{1}\right|^{p} \mathrm{~d} x\right)^{1 / p} \\
& \lesssim|t|\left(\frac{1}{r^{\lambda}} \int_{B(s, r)}\left(\int_{\left|y_{1}\right|<\frac{|t|}{\xi}} \int_{\left|y_{2}\right|>\frac{|t|}{\xi}} \frac{\left|f_{1}\left(x-y_{1}\right)\right|\left|f_{2}\left(x-y_{2}\right)\right|}{\left(\left|y_{1}\right|+\left|y_{2}\right|\right)^{2 n}} \mathrm{~d} y_{1} \mathrm{~d} y_{2}\right)^{p} \mathrm{~d} x\right)^{1 / p} \\
& \quad+|t|\left\|T^{*}\right\|_{p, \lambda} \\
& \lesssim|t| \int_{\left|y_{1}\right|<\frac{|t|}{\xi}} \int_{\left|y_{2}\right|>\frac{|t|}{\xi}}\left(\frac{1}{r^{\lambda}} \int_{B(s, r)}\left|f_{1}\left(x-y_{1}\right)\right|^{p}\left|f_{2}\left(x-y_{2}\right)\right|^{p} \mathrm{~d} x\right)^{1 / p} \frac{1}{\left(\left|y_{1}\right|+\left|y_{2}\right|\right)^{2 n}} \mathrm{~d} y_{2} \\
& \quad+|t||| f_{1}\left\|_{p_{1}, \lambda_{1}}|| f_{2}\right\|_{p_{2}, \lambda_{2}} \\
& \lesssim|t||| f_{1}\left\|_{p_{1}, \lambda_{1}}\right\| f_{2} \|_{p_{2}, \lambda_{2}} \int_{\left|y_{1}\right|<\frac{|t|}{\xi}} \int_{\left|y_{2}\right|>\frac{|t|}{\xi}} \frac{1}{\left(\left|y_{1}\right|+\left|y_{2}\right|\right)^{2 n}} \mathrm{~d} y_{1} \mathrm{~d} y_{2}+|t| \\
& \lesssim|t| \int_{\left|y_{1}\right|<\frac{|t|}{\xi}} \frac{1}{\left|y_{1}\right|^{n-1}} \mathrm{~d} y_{1} \int_{\left|y_{2}\right|>\frac{|t|}{\xi}} \frac{1}{\left|y_{2}\right|^{n+1}} \mathrm{~d} y_{2}+|t| \\
& \lesssim|t| .
\end{aligned}
$$

We obtain

$$
\left\|I_{1}\right\|_{p, \lambda} \leq C|t|
$$

Now, we estimate $I_{2}$ using some ideas of [9], notice that

$$
\begin{aligned}
I_{2} \leq \sup _{\delta>0} \mid \iiint_{\begin{array}{c}
\min \left(\left|x-y_{1}\right|,\left|x-y_{2}\right|\right)>\frac{|t|}{\xi} \\
\left|x-y_{1}\right|^{2}+\left|x-y_{2}\right|^{2}>\delta \\
\left|x+t-y_{1}\right|^{2}+\left|x+t-y_{2}\right|^{2}>\delta
\end{array}}\left(K\left(x, y_{1}, y_{2}\right)-K\left(x+t, y_{1}, y_{2}\right)\right) \\
\quad \times\left(b\left(y_{1}\right)-b(x+t)\right) f_{1}\left(y_{1}\right) f_{2}\left(y_{2}\right) \mathrm{d} y_{1} \mathrm{~d} y_{2} \mid
\end{aligned}
$$




$$
\begin{aligned}
& +\sup _{\delta>0}\left|\iint_{\substack{\min \left(\left|x-y_{1}\right|,\left|x-y_{2}\right|\right)>\frac{|t|}{\xi} \\
\left|x-y_{1}\right|^{2}+\left|x-y_{2}\right|^{2}>\delta \\
\left|x+t-y_{1}\right|^{2}+\left|x+t-y_{2}\right|^{2}<\delta}} K\left(x, y_{1}, y_{2}\right)\left(b\left(y_{1}\right)-b(x+t)\right) \prod_{i=1}^{2} f_{i}\left(y_{i}\right) \mathrm{d} \vec{y}\right| \\
& +\sup _{\delta>0}\left|\iint_{\substack{\min \left(\left|x-y_{1}\right|,\left|x-y_{2}\right|\right)>\frac{|t|}{\xi} \\
\left|x-y_{1}\right|^{2}+\left|x-y_{2}\right|^{2}<\delta \\
\left|x+t-y_{1}\right|^{2}+\left|x+t-y_{2}\right|^{2}>\delta}} K\left(x+t, y_{1}, y_{2}\right)\left(b\left(y_{1}\right)-b(x+t)\right) \prod_{i=1}^{2} f_{i}\left(y_{i}\right) \mathrm{d} \vec{y}\right| \\
& =I_{2}^{1}+I_{2}^{2}+I_{2}^{3 .}
\end{aligned}
$$

In order to estimate $I_{2}^{1}$, by a consequence of condition (1.4), we get

$$
\left|K\left(x, y_{1}, y_{2}\right)-K\left(x^{\prime}, y_{1}, y_{2}\right)\right| \leq \frac{D\left|x-x^{\prime}\right|^{\gamma}}{\left(\left|x-y_{1}\right|+\left|x-y_{2}\right|\right)^{2 n+\gamma}}
$$

when $\left|x-x^{\prime}\right| \leq \frac{1}{8} \min \left\{\left|x-y_{1}\right|,\left|x-y_{2}\right|\right\}$. Then

$$
\begin{aligned}
I_{2}^{1} & \lesssim\|b\|_{L^{\infty}} \iint_{\min \left(\left|x-y_{1}\right|,\left|x-y_{2}\right|\right)>\frac{|t|}{\xi}} \frac{|t|^{\gamma}}{\left(\left|x-y_{1}\right|+\left|x-y_{2}\right|\right)^{2 n+\gamma}}\left|f_{1}\left(y_{1}\right)\right|\left|f_{2}\left(y_{2}\right)\right| \mathrm{d} \vec{y} \\
& \lesssim|t|^{\gamma} \int_{\left|y_{1}\right|>\frac{|t|}{\xi}} \int_{\left|y_{2}\right|>\frac{|t|}{\xi}} \frac{\left|f_{1}\left(x-y_{1}\right)\right|\left|f_{2}\left(x-y_{2}\right)\right|}{\left(\left|y_{1}\right|+\left|y_{2}\right|\right)^{2 n+\gamma}} \mathrm{d} y_{1} \mathrm{~d} y_{2} .
\end{aligned}
$$

Hence, for every $s \in \mathbb{R}^{n}$ and $r>0$, Hölder's inequality and Minkowski's inequality give that

$$
\begin{aligned}
& \left(\frac{1}{r^{\lambda}} \int_{B(s, r)}\left|I_{2}^{1}\right|^{p} \mathrm{~d} x\right)^{1 / p} \\
& \lesssim|t|^{\gamma}\left(\frac{1}{r^{\lambda}} \int_{B(s, r)}\left(\int_{\left|y_{1}\right|>\frac{|t|}{\xi}} \int_{\left|y_{2}\right|>\frac{|t|}{\xi}} \frac{\left|f_{1}\left(x-y_{1}\right)\right|\left|f_{2}\left(x-y_{2}\right)\right|}{\left(\left|y_{1}\right|+\left|y_{2}\right|\right)^{2 n+\gamma}} \mathrm{d} y_{1} \mathrm{~d} y_{2}\right)^{p} \mathrm{~d} x\right)^{1 / p} \\
& \lesssim|t|^{\gamma} \int_{\left|y_{1}\right|>\frac{|t|}{\xi}} \int_{\left|y_{2}\right|>\frac{|t|}{\xi}}\left(\frac{1}{r^{\lambda}} \int_{B(s, r)}\left|f_{1}\left(x-y_{1}\right)\right|^{p}\left|f_{2}\left(x-y_{2}\right)\right|^{p} \mathrm{~d} x\right)^{1 / p} \\
& \quad \times \frac{1}{\left(\left|y_{1}\right|+\left|y_{2}\right|\right)^{2 n+\gamma}} \mathrm{d} y_{1} \mathrm{~d} y_{2} \\
& \lesssim|t|^{\gamma}\left\|f_{1}\right\|_{p_{1}, \lambda_{1}}|| f_{2} \|_{p_{2}, \lambda_{2}} \int_{\left|y_{1}\right|>\frac{|t|}{\xi}} \int_{\left|y_{2}\right|>\frac{|t|}{\xi}} \frac{1}{\left(\left|y_{1}\right|+\left|y_{2}\right|\right)^{2 n+\gamma}} \mathrm{d} y_{1} \mathrm{~d} y_{2} \\
& \lesssim|t|^{\gamma} \int_{\left|y_{1}\right|>\frac{|t|}{\xi}} \frac{1}{\left|y_{1}\right|^{n+\gamma / 2}} \mathrm{~d} y_{1} \int_{\left|y_{2}\right|>\frac{|t|}{\xi}} \frac{1}{\left|y_{2}\right|^{n+\gamma / 2}} \mathrm{~d} y_{2} \\
& \lesssim \xi^{\gamma} .
\end{aligned}
$$

Therefore,

$$
\left\|I_{2}^{1}\right\|_{p, \lambda} \leq C \xi^{\gamma}
$$


For $I_{2}^{2}$, as $\min \left(\left|x-y_{1}\right|,\left|x-y_{2}\right|\right)>\frac{|t|}{\xi},\left|x+t-y_{1}\right|^{2}+\left|x+t-y_{2}\right|^{2}<\delta$, we have $\left|x-y_{1}\right|^{2}+\left|x-y_{2}\right|^{2} \leq \frac{1}{(1-\xi)^{2}}\left(\left|x+t-y_{1}\right|^{2}+\left|x+t-y_{2}\right|^{2}\right) \leq \frac{\delta}{(1-\xi)^{2}}$. It follows that

$$
\begin{aligned}
I_{2}^{2} & \lesssim\|b\|_{L^{\infty}} \sup _{\delta>0} \iint_{\delta<\left|x-y_{1}\right|^{2}+\left|x-y_{2}\right|^{2}<\frac{\delta}{(1-\xi)^{2}}} \frac{\left|f_{1}\left(y_{1}\right)\right|\left|f_{2}\left(y_{2}\right)\right|}{\left(\left|x-y_{1}\right|+\left|x-y_{2}\right|\right)^{2 n}} \mathrm{~d} y_{1} \mathrm{~d} y_{2} \\
& \lesssim \sup _{\delta>0} \iint_{\delta<\left|y_{1}\right|^{2}+\left|y_{2}\right|^{2}<\frac{\delta}{(1-\xi)^{2}}} \frac{\left|f_{1}\left(x-y_{1}\right)\right|\left|f_{2}\left(x-y_{2}\right)\right|}{\left(\left|y_{1}\right|+\left|y_{2}\right|\right)^{2 n}} \mathrm{~d} y_{1} \mathrm{~d} y_{2} .
\end{aligned}
$$

Thus, for every $s \in \mathbb{R}^{n}$ and $r>0$, by Hölder's inequality and Minkowski's inequality we obtain

$$
\begin{aligned}
& \left(\frac{1}{r^{\lambda}} \int_{B(s, r)}\left|I_{2}^{2}\right|^{p} \mathrm{~d} x\right)^{1 / p} \\
& \lesssim \sup _{\delta>0}\left(\frac{1}{r^{\lambda}} \int_{B(s, r)}\left(\iint_{\delta<\left|y_{1}\right|^{2}+\left|y_{2}\right|^{2}<\frac{\delta}{(1-\xi)^{2}}} \frac{\left|f_{1}\left(x-y_{1}\right)\right|\left|f_{2}\left(x-y_{2}\right)\right|}{\left(\left|y_{1}\right|+\left|y_{2}\right|\right)^{2 n}} \mathrm{~d} \vec{y}\right)^{p} \mathrm{~d} x\right)^{1 / p} \\
& \lesssim \sup _{\delta>0} \iint_{\delta<\left|y_{1}\right|^{2}+\left|y_{2}\right|^{2}<\frac{\delta}{(1-\xi)^{2}}}\left(\frac{1}{r^{\lambda}} \int_{B(s, r)}\left|f_{1}\left(x-y_{1}\right)\right|^{p}\left|f_{2}\left(x-y_{2}\right)\right|^{p} \mathrm{~d} x\right)^{1 / p} \\
& \quad \times \frac{1}{\left(\left|y_{1}\right|+\left|y_{2}\right|\right)^{2 n}} \mathrm{~d} \vec{y} \\
& \lesssim\left\|f_{1}\right\|_{p_{1}, \lambda_{1}}|| f_{2} \|_{p_{2}, \lambda_{2}} \sup _{\delta>0} \iint_{\delta<\left|y_{1}\right|^{2}+\left|y_{2}\right|^{2}<\frac{\delta}{(1-\xi)^{2}}} \frac{1}{\left(\left|y_{1}\right|+\left|y_{1}\right|\right)^{2 n}} \mathrm{~d} y_{2} \\
& \lesssim \sup _{\delta>0} \int_{\left|y_{2}\right|^{2} \leq \delta} \int_{\delta-\left|y_{2}\right|^{2}<\left|y_{1}\right|^{2}<\delta /(1-\xi)^{2}-\left|y_{2}\right|^{2}} \frac{1}{\left(\left|y_{1}\right|+\left|y_{2}\right|\right)^{2 n}} \mathrm{~d} y_{1} \mathrm{~d} y_{2} \\
& +\sup _{\delta>0} \int_{\delta \leq\left|y_{2}\right|^{2} \leq \delta /(1-\xi)^{2}} \int_{\left|y_{1}\right|^{2} \leq \delta /(1-\xi)^{2}-\left|y_{2}\right|^{2}} \frac{1}{\left(\left|y_{1}\right|+\left|y_{2}\right|\right)^{2 n}} \mathrm{~d} y_{1} \mathrm{~d} y_{2} \\
& \lesssim(1-\xi)^{-n}-1 \lesssim \xi .
\end{aligned}
$$

We have

$$
\left\|I_{2}^{2}\right\|_{p, \lambda} \leq C \xi
$$

For $I_{2}^{3}$, we proceed in a similar way. It is easy to find that $\min \left(\left|x-y_{1}\right|,\left|x-y_{2}\right|\right)>$ $\frac{|t|}{\xi},\left|x+t-y_{1}\right|^{2}+\left|x+t-y_{2}\right|^{2}>\delta$, then we have $\frac{\delta}{(1+\xi)^{2}} \leq\left|x-y_{1}\right|^{2}+\left|x-y_{2}\right|^{2} \leq \delta$. Hence,

$$
\begin{aligned}
I_{2}^{3} & \lesssim\|b\|_{L^{\infty}} \sup _{\delta>0} \iint_{\delta /(1+\xi)^{2} \leq\left|x-y_{1}\right|^{2}+\left|x-y_{2}\right|^{2} \leq \delta} \frac{\left|f_{1}\left(y_{1}\right)\right|\left|f_{2}\left(y_{2}\right)\right|}{\left(\left|x-y_{1}\right|+\left|x-y_{2}\right|\right)^{2 n}} \mathrm{~d} y_{1} \mathrm{~d} y_{2} \\
& \lesssim \sup _{\delta>0} \iint_{\delta /(1+\xi)^{2} \leq\left|y_{1}\right|^{2}+\left|y_{2}\right|^{2} \leq \delta} \frac{\left|f_{1}\left(x-y_{1}\right)\right|\left|f_{2}\left(x-y_{2}\right)\right|}{\left(\left|y_{1}\right|+\left|y_{2}\right|\right)^{2 n}} \mathrm{~d} y_{1} \mathrm{~d} y_{2},
\end{aligned}
$$


consequently, for every $s \in \mathbb{R}^{n}$ and $r>0$, by Hölder's inequality and Minkowski's inequality we get

$$
\begin{aligned}
& \left(\frac{1}{r^{\lambda}} \int_{B(s, r)}\left|I_{2}^{3}\right|^{p} \mathrm{~d} x\right)^{1 / p} \\
& \lesssim \sup _{\delta>0}\left(\frac{1}{r^{\lambda}} \int_{B(s, r)}\left(\iint_{\delta /(1+\xi)^{2}<\left|y_{1}\right|^{2}+\left|y_{2}\right|^{2}<\delta} \frac{\left|f_{1}\left(x-y_{1}\right)\right|\left|f_{2}\left(x-y_{2}\right)\right|}{\left(\left|y_{1}\right|+\left|y_{2}\right|\right)^{2 n}} \mathrm{~d} \vec{y}\right)^{p} \mathrm{~d} x\right)^{1 / p} \\
& \lesssim \sup _{\delta>0}\left(\frac{1}{r^{\lambda}} \int_{B(s, r)}\left|f_{1}\left(x-y_{1}\right)\right|^{p}\left|f_{2}\left(x-y_{2}\right)\right|^{p} \mathrm{~d} x\right)^{1 / p} \\
& \quad \times \frac{1}{\left(\left|y_{1}\right|+\left|y_{2}\right|\right)^{2 n}} \mathrm{~d} \vec{y} \\
& \lesssim \sup _{\delta>0} \int_{\left|y_{2}\right|^{2} \leq \delta /(1+\xi)^{2}} \int_{\delta /(1+\xi)^{2}-\left|y_{2}\right|^{2}<\left|y_{1}\right|^{2}<\delta-\left|y_{2}\right|^{2}} \frac{1}{\left(\left|y_{1}\right|+\left|y_{2}\right|\right)^{2 n}} \mathrm{~d} y_{1} \mathrm{~d} y_{2} \\
& +\sup _{\delta>0} \int_{\delta /(1+\xi)^{2} \leq\left|y_{2}\right|^{2} \leq \delta} \int_{\left|y_{1}\right|^{2} \leq \delta-\left|y_{2}\right|^{2}<\delta} \frac{1}{\left(\left|y_{1}\right|+\left|y_{2}\right|\right)^{2 n}} \mathrm{~d} y_{1} \mathrm{~d} y_{2} \\
& \lesssim 1-(1+\xi)^{-n} \lesssim \xi .
\end{aligned}
$$

Therefore,

$$
\left\|I_{2}^{3}\right\|_{p, \lambda} \leq C \xi
$$

In summary,

$$
\left\|I_{2}\right\|_{p, \lambda} \leq C \xi
$$

Next, we estimate $I_{3}$

$$
\begin{aligned}
I_{3} \lesssim & \|\nabla b\|_{L^{\infty}} \int_{\left|x-y_{1}\right|<\frac{|t|}{\xi}} \int_{\left|x-y_{2}\right|>\frac{|t|}{\xi}} \frac{\left|f_{1}\left(y_{1}\right)\right|\left|f_{2}\left(y_{2}\right)\right|}{\left(\left|x-y_{1}\right|+\left|x-y_{2}\right|\right)^{2 n-1}} \mathrm{~d} y_{1} \mathrm{~d} y_{2} \\
& +\|\nabla b\|_{L^{\infty}} \int_{\left|x-y_{1}\right|>\frac{|t|}{\xi}} \int_{\left|x-y_{2}\right|<\frac{|t|}{\xi}} \frac{\left|f_{1}\left(y_{1}\right)\right|\left|f_{2}\left(y_{2}\right)\right|}{\left(\left|x-y_{1}\right|+\left|x-y_{2}\right|\right)^{2 n-1}} \mathrm{~d} y_{1} \mathrm{~d} y_{2} \\
\lesssim & \int_{\left|y_{1}\right|<\frac{|t|}{\xi}} \int_{\left|y_{2}\right|>\frac{|t|}{\xi}} \frac{\left|f_{1}\left(x-y_{1}\right)\right|\left|f_{2}\left(x-y_{2}\right)\right|}{\left(\left|y_{1}\right|+\left|y_{2}\right|\right)^{2 n-1}} \mathrm{~d} y_{1} \mathrm{~d} y_{2},
\end{aligned}
$$

provided $(\delta / 2)^{1 / 2}>|t| / \xi$, then for every $s \in \mathbb{R}^{n}$ and $r>0$, Hölder's inequality and Minkowski's inequality give that

$$
\begin{aligned}
& \left(\frac{1}{r^{\lambda}} \int_{B(s, r)}\left|I_{3}\right|^{p} \mathrm{~d} x\right)^{1 / p} \\
& \lesssim\left(\frac{1}{r^{\lambda}} \int_{B(s, r)}\left(\int_{\left|y_{1}\right|<\frac{|t|}{\xi}} \int_{\left|y_{2}\right|>\frac{|t|}{\xi}} \frac{\left|f_{1}\left(x-y_{1}\right)\right|\left|f_{2}\left(x-y_{2}\right)\right|}{\left(\left|y_{1}\right|+\left|y_{2}\right|\right)^{2 n-1}} \mathrm{~d} y_{1} \mathrm{~d} y_{2}\right)^{p} \mathrm{~d} x\right)^{1 / p} \\
& \lesssim \int_{\left|y_{1}\right|<\frac{|t|}{\xi}} \int_{\left|y_{2}\right|>\frac{|t|}{\xi}}\left(\frac{1}{r^{\lambda}} \int_{B(s, r)}\left|f_{1}\left(x-y_{1}\right)\right|^{p}\left|f_{2}\left(x-y_{2}\right)\right|^{p} \mathrm{~d} x\right)^{1 / p} \frac{\mathrm{d} y_{1} \mathrm{~d} y_{2}}{\left(\left|y_{1}\right|+\left|y_{2}\right|\right)^{2 n-1}} \\
& \lesssim|t| / \xi .
\end{aligned}
$$


We get

$$
\left\|I_{3}\right\|_{p, \lambda} \leq C|t| / \xi
$$

Finally, for the last part $I_{4}$, we estimate it as $I_{3}$. By replacing the region of integration $\left\{\left(x, y_{1}, y_{2}\right): \min \left(\left|x-y_{1}\right|,\left|x-y_{2}\right|\right)<|t| / \xi\right\}$ with a larger one $\left\{\left(x, y_{1}, y_{2}\right): \min \left(\left|x+t-y_{1}\right|,\left|x+t-y_{2}\right|\right)<|t| / \xi+|t|\right\}$. We know that

$$
\begin{aligned}
I_{4} \lesssim & \|\nabla b\|_{L^{\infty}} \int_{\left|x+t-y_{1}\right|<\frac{|t|}{\xi}+|t|} \int_{\left|x+t-y_{2}\right|>\frac{|t|}{\xi}} \frac{\left|f_{1}\left(y_{1}\right)\right|\left|f_{2}\left(y_{2}\right)\right|}{\left(\left|x+t-y_{1}\right|+\left|x+t-y_{2}\right|\right)^{2 n-1}} \mathrm{~d} \vec{y} \\
& +\|\nabla b\|_{L^{\infty}} \int_{\left|x+t-y_{1}\right|>\frac{|t|}{\xi}} \int_{\left|x+t-y_{2}\right|<\frac{|t|}{\xi}+|t|} \frac{\left|f_{1}\left(y_{1}\right)\right|\left|f_{2}\left(y_{2}\right)\right|}{\left(\left|x+t-y_{1}\right|+\left|x+t-y_{2}\right|\right)^{2 n-1}} \mathrm{~d} \vec{y} \\
& \lesssim \int_{\left|y_{1}\right|<\frac{|t|}{\xi}+|t|} \int_{\left|y_{2}\right|>\frac{|t|}{\xi}} \frac{\left|f_{1}\left(x+t-y_{1}\right)\right|\left|f_{2}\left(x+t-y_{2}\right)\right|}{\left(\left|y_{1}\right|+\left|y_{2}\right|\right)^{2 n-1}} \mathrm{~d} \vec{y},
\end{aligned}
$$

provided $(\delta / 2)^{1 / 2}>|t| / \xi$, then for every $s \in \mathbb{R}^{n}$ and $r>0$,

$$
\begin{aligned}
& \left(\frac{1}{r^{\lambda}} \int_{B(s, r)}\left|I_{4}\right|^{p} \mathrm{~d} x\right)^{1 / p} \\
& \lesssim\left(\frac{1}{r^{\lambda}} \int_{B(s, r)}\left(\int_{\left|y_{1}\right|<\frac{|t|}{\xi}+|t|} \int_{\left|y_{2}\right|>\frac{|t|}{\xi}} \frac{\left|f_{1}\left(x+t-y_{1}\right)\right|\left|f_{2}\left(x+t-y_{2}\right)\right|}{\left(\left|y_{1}\right|+\left|y_{2}\right|\right)^{2 n-1}} \mathrm{~d} \vec{y}\right)^{p} \mathrm{~d} x\right)^{1 / p} \\
& \lesssim \int_{\left|y_{1}\right|<\frac{|t|}{\xi}+|t|} \int_{\left|y_{2}\right|>\frac{|t|}{\xi}}\left(\frac{1}{r^{\lambda}} \int_{B(s, r)}\left|f_{1}\left(x+t-y_{1}\right)\right|^{p}\left|f_{2}\left(x+t-y_{2}\right)\right|^{p} \mathrm{~d} x\right)^{1 / p} \\
& \quad \times \frac{1}{\left(\left|y_{1}\right|+\left|y_{2}\right|\right)^{2 n-1}} \mathrm{~d} y_{1} \mathrm{~d} y_{2} \\
& \lesssim\left\|f_{1}\right\|_{p_{1}, \lambda_{1}}|| f_{2} \|_{p_{2}, \lambda_{2}} \int_{\left|y_{1}\right|<\frac{|t|}{\xi}+|t|} \frac{1}{\left|y_{1}\right|^{n-2}} \mathrm{~d} y_{1} \int_{\left|y_{2}\right|>\frac{|t|}{\xi}} \frac{1}{\left|y_{2}\right|^{n+1}} \mathrm{~d} y_{2} \\
& \lesssim|t| / \xi+|t| .
\end{aligned}
$$

Consequently,

$$
\left\|I_{4}\right\|_{p, \lambda} \leq C|t| / \xi+|t| .
$$

Then inequalities (3.3), (3.4), (3.5) and (3.6) imply (3.2), and in this way, we can conclude that $T_{b, 1}^{*}$ is compact. By symmetry, $T_{b, 2}^{*}$ is also compact.

Acknowledgement. This research was supported by the NNSF of China (Grant No. 11271330) and the NSF of Henan Province (Grant No. 122300410117).

\section{REFERENCES}

1. A. Alphonse, An end point estimates for maximal commutators, J. Fourier Anal. Appl. 6 (2000), 449-456.

2. Á. Bényi and R.H. Torres, Compact bilinear operators and commutators, Proc. Amer. Math. Soc. 141 (2013), no. 10, 3609-3621.

3. R. Bu and J. Chen, The compactness of the commutator of multilinear singular integral operators with non-smooth kernels, arXiv:1411.1905v1. 
4. A.P. Calderón, Intermediate spaces and interpolation, the complex method, Studia Math. 24 (1964), 113-190.

5. J. Chen and Y. Zhang, Weighted endpoint estimates for maximal commutators associated with the sections, Acta. Math. Sin. (Engl. Ser.). 26 (2010), 1463-1474.

6. Y. Chen, Y. Ding and X. Wang, Compactness of commutators for singular integrals on Morrey spaces, Canad. J. Math. 64 (2012), no. 2, 257-281.

7. R.R. Coifman and Y. Meyer, On commutators of singular integrals and bilinear singular integrals, Trans. Amer. Math. Soc. 212 (1975),315-331.

8. R.R. Coifman and R. Rochberg, Another characterization of BMO, Proc. Amer. Math. Soc. 79 (1980), 249-254.

9. Y. Ding, T. Mei and Q. Xue, Compactness of maximal commutators of bilinear CalderónZygmund singular integral operators, arXiv:1310.5787v3.

10. Y. Ding and T. Mei, Boundedness and compactness for the commutators of bilinear operators on Morrey spaces., Potential Analysis, to appear.

11. X.T. Duong, R. Gong, L. Grafakos, J. Li and L. Yan, Maximal operator for multilinear singular integrals with non-smooth kernels, Indiana Univ. Math. J. 58 (2009), 2517-2541.

12. X.T. Duong, L. Grafakos and L. Yan, Multilinear operators with non-smooth kernels and commutators of singular integrals, Trans. Amer. Math. Soc. 362 (2010), 2089-2113.

13. X.T. Duong and A. MaIntosh, Singular integral operators with non-smooth kernels on irregular domains, Rev. Mat. Iberoamericana 15 (1999),233-265.

14. C. Fefferman and E.M. Stein, $H^{p}$ spaces of several variables, Acta Math. 129 (1972), 137193.

15. L. Grafakos, L Liu. and D. Yang, Multiple-weighted norm inequalities for maximal multilinear singular integrals with non-smooth kernels, Proc. Roy. Soc. Edinburgh Sect. A, 141 (2011), 755-775.

16. L. Grafakos and R.H. Torres, Maximal operator and weighted norm inequalities for multilinear singular integrals, Indiana Univ. Math. J. 51 (2002), 1261-1276.

17. L. Grafakos and R.H. Torres, Multilinear Calderón-Zygmund theory, Adv. Math. 165 (2002), 124-164.

18. G. Hu and D. Yang, Weighted estimates for singular integral operators with nonsmooth kernels and applications, J. Aust. Math. Soc. 85 (2008), 377-417.

19. G. Hu and Y. Zhu, Weighted norm inequality with general weights for the commutator of Calderón, Acta. Math. Sin. (Engl. Ser.). 29 (2013), 505-514.

20. F. John and L. Nirenberg, On functions of bounded mean oscillation, Comm. Pure Appl. Math. 14 (1961), 415-426.

21. A. Lerner, S. Ombrosi, C. Pérez, R.H. Torres and R. Trujillo-González, New maximal functions and multiple weights for the multilinear Calderón-Zygmund theory, Adv. Math. 220 (2009), 1222-1264.

22. C. Morrey, On the solutions of quasi linear elleptic partial diferential equations, Trans. Amer. Math. Soc. 43 (1938), 126-166.

23. C. Pérez, Endpoint estimates for commutators of singular integral operators, J. Funct. Anal. 128 (1995), 163-185.

24. C. Pérez, G. Pradolini, R.H. Torres and R. Trujillo-González, End-points estimates for iterated commutators of multilinear singular integrals, Bull. London Math. Soc. 46 (2014), $26-42$.

25. L.E. Persson, M.A. Ragusa, N. Samko and P. Wall, Commutators of Hardy operators in vanishing Morrey spaces, AIP Conference Proceedings, 1493, 859-866;

26. M.A. Ragusa, Homogeneous Herz spaces and regularity results, Nonlinear Anal. 71 (2009), no. 12, e1909-e1914.

27. A. Uchiyama, On the compactness of operators of Hankel type, Tôhoku Math. J. 30 (1978), no. $1,163-171$.

28. Q. Xue, Weighted estimates for the iterated commutators of multilinear maximal and fractional type operators, Studia Math. 217 (2013), no. 2, 97-122. 
1 Department of Mathematics, Zhejiang University, Hangzhou 310027, P.R. ChInA.

E-mail address: burui0@163.com

E-mail address: huangqiang0704@163.com

2 Department of Mathematics and Information Science, Zhengzhou University of Light Industry, Zhengzhou 450002, P.R. China.

E-mail address: xlguo@zzuli.edu.cn 